\title{
Environmental Light Is Required for Maintenance of Long-Term Memory in Drosophila
}

\author{
Show Inami, ${ }^{1}$ Shoma Sato, ${ }^{1}$ Shu Kondo, ${ }^{2}$ Hiromu Tanimoto, ${ }^{3}$ ๑Toshihiro Kitamoto,, 5 and ${ }^{4}$ Takaomi Sakai ${ }^{1}$ \\ ${ }^{1}$ Department of Biological Sciences, Tokyo Metropolitan University, Tokyo 192-0397, Japan, ${ }^{2}$ Genetic Strains Research Center, National Institute of \\ Genetics, Shizuoka 411-8540, Japan, ${ }^{3}$ Tohoku University Graduate School of Life Sciences, Sendai 980-8577, Japan, ${ }^{4}$ Department of Anesthesia and \\ Pharmacology, Carver College of Medicine, and 5 Interdisciplinary Programs in Genetics and Neuroscience, University of Iowa, Iowa City, Iowa 52242
}

\begin{abstract}
Long-term memory (LTM) is stored as functional modifications of relevant neural circuits in the brain. A large body of evidence indicates that the initial establishment of such modifications through the process known as memory consolidation requires learning-dependent transcriptional activation and de novo protein synthesis. However, it remains poorly understood how the consolidated memory is maintained for a long period in the brain, despite constant turnover of molecular substrates. Using the Drosophila courtship conditioning assay of adult males as a memory paradigm, here, we show that in Drosophila, environmental light plays a critical role in LTM maintenance. LTM is impaired when flies are kept in constant darkness (DD) during the memory maintenance phase. Because light activates the brain neurons expressing the neuropeptide pigment-dispersing factor (Pdf), we examined the possible involvement of Pdf neurons in LTM maintenance. Temporal activation of Pdf neurons compensated for the DD-dependent LTM impairment, whereas temporal knockdown of Pdf during the memory maintenance phase impaired LTM in light/dark cycles. Furthermore, we demonstrated that the transcription factor cAMP response element-binding protein (CREB) is required in the memory center, namely, the mushroom bodies (MBs), for LTM maintenance, and Pdf signaling regulates light-dependent transcription via CREB. Our results demonstrate for the first time that universally available environmental light plays a critical role in LTM maintenance by activating the evolutionarily conserved memory modulator CREB in MBs via the Pdf signaling pathway.
\end{abstract}

Key words: clock neurons; CREB; Drosophila; long-term memory; memory maintenance; pigment-dispersing factor

Significance Statement

Temporary memory can be consolidated into long-term memory (LTM) through de novo protein synthesis and functional modifications of neuronal circuits in the brain. Once established, LTM requires continual maintenance so that it is kept for an extended period against molecular turnover and cellular reorganization that may disrupt memory traces. How is LTM maintained mechanistically? Despite the critical importance of LTM maintenance, its molecular and cellular underpinnings remain elusive. This study using Drosophila is significant because it revealed for the first time in any organism that universally available environmental light plays an essential role in LTM maintenance. Interestingly, light does so by activating the evolutionarily conserved transcription factor cAMP response element-binding protein via peptidergic signaling.

\section{Introduction}

A newly formed memory is initially labile, but under certain circumstances, it is consolidated into a more stable long-term memory (LTM). Previous studies using various animal species have

\footnotetext{
Received June 3, 2019; revised Nov. 14, 2019; accepted Dec. 12, 2019.

Author contributions: S.I., T.K., and T.S. designed research; S.I. and S.S. performed research; S.I., S.S., and T.S. analyzed data; S.I., H.T., T.K., T.S., S.K., T.K., and T.S. wrote the paper.

This work was supported by JSPS KAKENHI Grants (18H04887 and 16H04816 to T.S., 17H01378, 17H05545, 16H01496, and 26250001 to H.T., and 15J06303 to S.I.). We thank Yuto Kurata and Yushun Sato for technical assistance, and Toshiro Aigaki for helpful discussions.

The authors declare no competing financial interests.

Correspondence should be addressed to Takaomi Sakai at sakai-takaomi@tmu.ac.jp.

https://doi.org/10.1523/JNEUROSCI.1282-19.2019

Copyright $\odot 2020$ the authors
}

shown that activation of specific transcription factors, such as the cAMP response element-binding protein (CREB), and the corresponding de novo protein synthesis are essential for memory consolidation (Yin and Tully, 1996; Y. S. Lee et al., 2008; Kandel, 2012). Once consolidated, LTM requires continual maintenance for its long-term storage and subsequent recall, because memory traces gradually decay because of molecular turnover and cellular reorganization. Similar to memory consolidation, transcriptional activation and de novo protein synthesis are required for LTM maintenance (Bekinschtein et al., 2007; Alberini, 2009; Majumdar et al., 2012; Fioriti et al., 2015; Hirano et al., 2016). For example, the maintenance of hippocampal long-term potentiation (LTP) and spatial memory in mice is dependent on the 
prion-like translational regulator CPEB3 (Fioriti et al., 2015). Furthermore, transcriptional regulation through CREB and its coactivator CRTC plays crucial roles in LTM maintenance in the Drosophila memory center, namely, the mushroom bodies (MBs; Hirano et al., 2016), suggesting that memory consolidation and maintenance share some of the same molecular mechanisms.

In Drosophila, aversive olfactory memory consists of genetically distinct memory components (Margulies et al., 2005). One of the components, LTM, lasts $>1 \mathrm{~d}$, and it is CREB- and de novo protein synthesis-dependent (Margulies et al., 2005; Davis, 2011; Dubnau and Chiang, 2013). Inhibition of protein synthesis or induction of a CREB repressor attenuates $1 \mathrm{~d}$ memory (Tully et al., 1994; Yin et al., 1994). On the basis of these findings, it is generally believed that memory consolidation completes within $1 \mathrm{~d}$ after conditioning (Margulies et al., 2005; Davis, 2011). Thus, in Drosophila, the LTM maintenance phase is conceptually defined on the basis of an empirical justification as the time after LTM is fully formed and consolidated (from $1 \mathrm{~d}$ after conditioning). An obvious and important question is how transcriptional activation and the following protein synthesis are triggered during the memory maintenance phase. Unlike transcriptional and translational activations involved in memory consolidation, those associated with LTM maintenance cannot be directly controlled by stimuli that induce memory formation, because there is a significant time separation between stimulus-induced memory formation and the memory maintenance process, and LTM should be continually maintained. However, the molecular and cellular underpinnings of active LTM maintenance, which is transcription- and translation-dependent, still remain elusive.

Earth's rotation generates the daily cycle of day and night, and the rhythmic light/dark (LD) cycles have a significant impact on animal behavior and physiology. In animals, light is not only essential for acquiring information for image-forming vision in nature but also acts as a powerful modulator of brain functions such as circadian entrainment, hormone secretion, sleep-wake cycles, mood, and cognitive functions (Altimus et al., 2008; Vandewalle et al., 2009; Crocker and Sehgal, 2010; LeGates et al., 2012). Using the diurnal fruit fly Drosophila melanogaster, here, we found that LTM was severely impaired in flies kept in constant darkness (DD) after memory consolidation. Thus, LTM maintenance is found to be light-dependent. In Drosophila, light activates photoreceptors in the brain neurons expressing the pigment-dispersing factor ( $\mathrm{Pdf}$ ), a neuropeptide, and increases their spontaneous firing rate (Sheeba et al., 2008; Fogle et al., 2011; Ni et al., 2017). Considering the physiological properties of Pdf neurons, it is possible that those neurons regulate lightdependent LTM maintenance. In this study, we found that the Pdf neurons play an essential role in light-dependent LTM maintenance. Our results demonstrate for the first time that environmental light, which is available daily to all animals under normal conditions, plays a critical role in LTM maintenance by reactivating the evolutionarily conserved memory modulator CREB via Pdf signaling.

\section{Materials and Methods}

Fly stocks. All flies were raised on glucose-yeast-cornmeal medium in $12 \mathrm{~h}$ $\mathrm{LD}$ cycles at $25.0 \pm 0.5^{\circ} \mathrm{C}(45-60 \%$ relative humidity). Virgin males and females were collected without anesthesia within $8 \mathrm{~h}$ after eclosion. The fly stocks used for this study were as follows: wild-type Canton-S (CS), $P d f^{01}$ (BL26654), Pdf ${ }^{5304}$ (BL33068), Pdf-GAL4 (BL6900), c929 (BL25373), R14F03 (BL48648), R18F07 (BL47876), R61G12-LexA (BL52685), R41C10 (BL50121), R55D03 (BL47656), R19B03 (BL49830), c305a (BL30829), UASFLP (BL4539), UAS-Kir2.1::eGFP (BL6596), UAS-TrpA1 (BL26263), UAS- $m C D 8:: G F P$ (BL5137), UAS-mCherry::NLS (BL38424), UAS-mCD8::RFP (BL32218), UAS-Pdf RNAi (VDRC4380), UAS-CrebB-B (also known as UAS-dCREB2- $b$, BL7219), tub-GAL80 ${ }^{\text {ts }}$ (BL7017), LexAop2-FLPL (BL55820), LexAop2-mCD8::GFP (BL32203), LexAop-TrpA1 (provided by Dr. Mani Ramaswami, Trinity College Institute of Neuroscience), hsCrebB-B (also known as hs-dCREB2-b; Yin et al., 1994; Sakai et al., 2004), UAS-Pdf (provided by Dr. Taishi Yoshii, Okayama University), UAS-luc RNAi (provided by Dr. Kanae Ando, Tokyo Metropolitan University), UAS $>$ STOP $>$ Kir 2.1::eGFP (provided by Dr. David J. Anderson, Caltech), and CRE $>m$ Cherry::STOP $>$ luc (provided by Dr. Jerry C. P. Yin, University of Wisconsin-Madison). All lines for behavior experiments except for $P d f^{o 1}$, $P d f r^{5304}$, UAS-luc RNAi, and LexAop2-FLPL were outcrossed for at least five generations to white ${ }^{1118}$ flies with the CS genetic background.

Courtship conditioning assay. The courtship conditioning assay was performed as previously described (Sakai et al., 2004) with some modifications. Unreceptive mated females were prepared as "trainers" $1 \mathrm{~d}$ before they were used for courtship conditioning. In this conditioning, a virgin CS female and a male (3-6 d old) were placed in an acrylic courtship chamber ( $15 \mathrm{~mm}$ diameter $\times 3 \mathrm{~mm}$ depth) for copulation. For LTM, a 3- to 5-d-old male was placed with a mated female (4-7 d old) in a conditioning chamber ( $15 \mathrm{~mm}$ diameter $\times 5 \mathrm{~mm}$ depth) containing food for $7 \mathrm{~h}$ either with (conditioned) or without (naive) a single premated female ( $7 \mathrm{~h}$ conditioning). If males re-mated with mated females during conditioning, we discarded such males after conditioning. After $7 \mathrm{~h}$ conditioning, only flies showing courtship behaviors toward the mated female but not copulating successfully were transferred to a glass tube with food ( $12 \mathrm{~mm}$ diameter $\times 75 \mathrm{~mm}$ depth) and kept in isolation for 1,2 , or $5 \mathrm{~d}$ until the test. The test was performed using a freeze-killed virgin female in a test chamber $(15 \mathrm{~mm}$ diameter $\times 3 \mathrm{~mm}$ depth $)$. All procedures in the experiments were performed at $25 \pm 1.0^{\circ} \mathrm{C}(45-60 \%$ relative humidity) except for the temperature shift experiments. Courtship index (CI) was used for quantifying male courtship behaviors of individual flies and was calculated manually. $\mathrm{CI}$ is defined as the percentage of time spent in performing courtship behaviors during a given observation period $(10 \mathrm{~min})$. We first measured $\mathrm{CI}$ in conditioned and naive males $\left(\mathrm{CI}_{\text {Conditioned }}\right.$ and $\mathrm{CI}_{\text {Naive }}$, respectively), and then mean $\mathrm{CI}_{\text {Naive }}$ and mean $\mathrm{CI}_{\text {Conditioned }}$ were calculated. To quantify courtship memory as previously reported (S. S. Lee et al., 2017), memory index (MI) was calculated using the following formula: $\mathrm{MI}=\left(\right.$ mean $\mathrm{CI}_{\text {Naive }}-$ mean $\mathrm{CI}_{\text {Conditioned }} / \mathrm{m}_{\text {mean }} \mathrm{CI}_{\text {Naive }}$.

Lighting conditions in courtship conditioning. When courtship conditioning was performed in a dark place, conditioning chambers were placed in a temperature-regulated $\left(25.0 \pm 0.5^{\circ} \mathrm{C}\right)$ light-tight incubator (MIR-254, Sanyo Electric). To check whether mating occurs during conditioning, we observed the flies for $10 \mathrm{~s}$ every $30 \mathrm{~min}$ by opening the incubator door. To determine whether lighting conditions affect memory maintenance, conditioned and naive flies were kept in a light-tight incubator (MIR-254, Sanyo Electric) for 1, 2, or $4 \mathrm{~d}$ after $7 \mathrm{~h}$ conditioning.

Temporal activation of Pdf neurons. The temperature-sensitive cation channel TrpAl was used to activate Pdf neurons (Hamada et al., 2008). For the activation of Pdf neurons for $2 \mathrm{~d}$ during DD (Days 2 and 3 after $7 \mathrm{~h}$ conditioning), $P d f$-GAL4/UAS-TrpA1 flies were kept at $30^{\circ} \mathrm{C}$ for $8 \mathrm{~h}$ within each subjective day [circadian time (CT) 0-8] or night (CT 1220). However, DD-dependent LTM impairment was not improved. Because it is possible that the activation of Pdf neurons under this condition is insufficient for LTM maintenance, the flies were kept at $34^{\circ} \mathrm{C}$ for $8 \mathrm{~h}$ within each subjective day (CT $0-8$ ) or night (CT 12-20). UAS-TrpA1/+ and $P d f$-GAL4/+ flies were used as the control.

Temporal gene expression using TARGET system. The tub-GAL80 ${ }^{\text {ts }}$ transgene used in the TARGET system (McGuire et al., 2003) encodes a ubiquitously expressed, temperature-sensitive GAL4 repressor that is active at the permissive temperature $\left(\mathrm{PT} ; 25^{\circ} \mathrm{C}\right)$ but not at the restrictive temperature (RT; 30 or $32^{\circ} \mathrm{C}$ ). By using UAS- $P d f$ RNAi combined with the TARGET system, we knocked down Pdf in GAL4-positive neurons at $\mathrm{RT}\left(30^{\circ} \mathrm{C}\right)$, but not at PT. In these experiments, we shifted PT to RT and vice versa during three experimental phases: $24 \mathrm{~h}$ before the end of conditioning, $48-72 \mathrm{~h}$ after conditioning, and $24 \mathrm{~h}$ before the initiation of the test. Furthermore, to drive the expression of a UAS-CrebB-B construct in MBs during a specific temporal phase, the TARGET system was 
A

$\begin{array}{llllll}\mathrm{D} 0 & \mathrm{D} 1 & \mathrm{D} 2 & \mathrm{D} 3 & \mathrm{D} 4 & \mathrm{D} 5\end{array}$

(1)

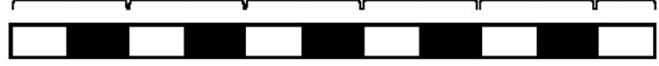

(2)

(3)

(4)

( 5 )

(6)

( 7 )

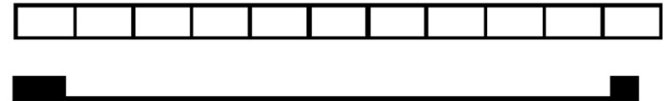

Conditioning

(7 h)

B

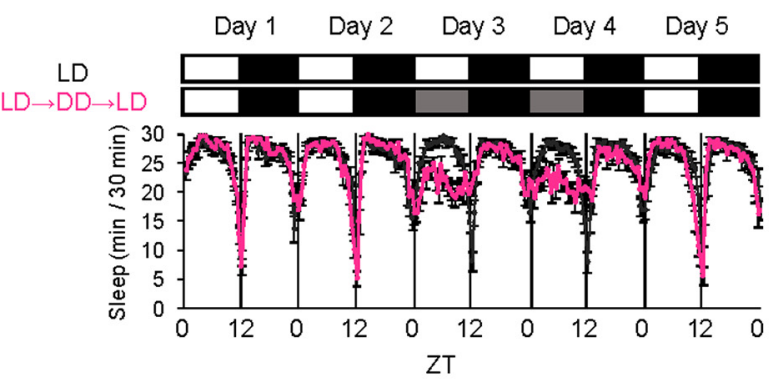

D

Naîve Conditioned

Day 0 Day 1

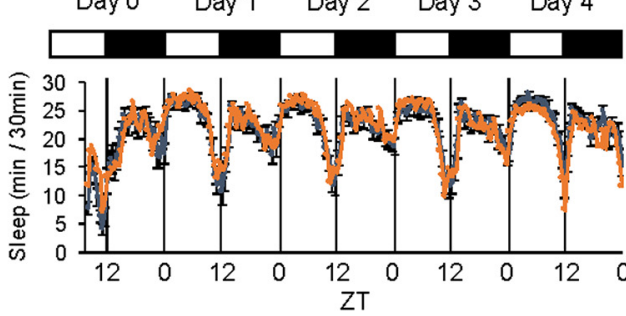

$\mathbf{F}$

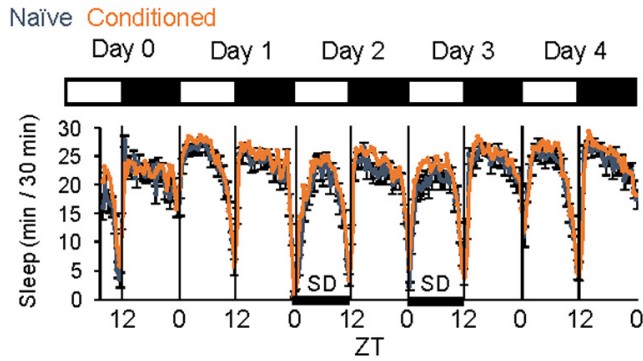

Test (10 $\mathrm{min})$
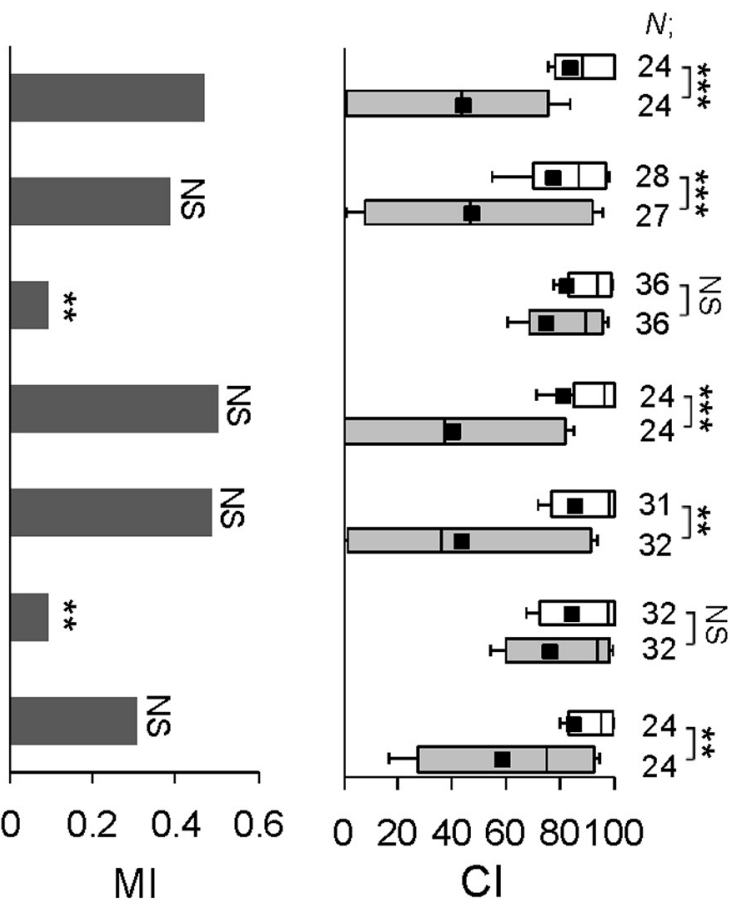

020406080100

$\mathrm{Cl}$

H
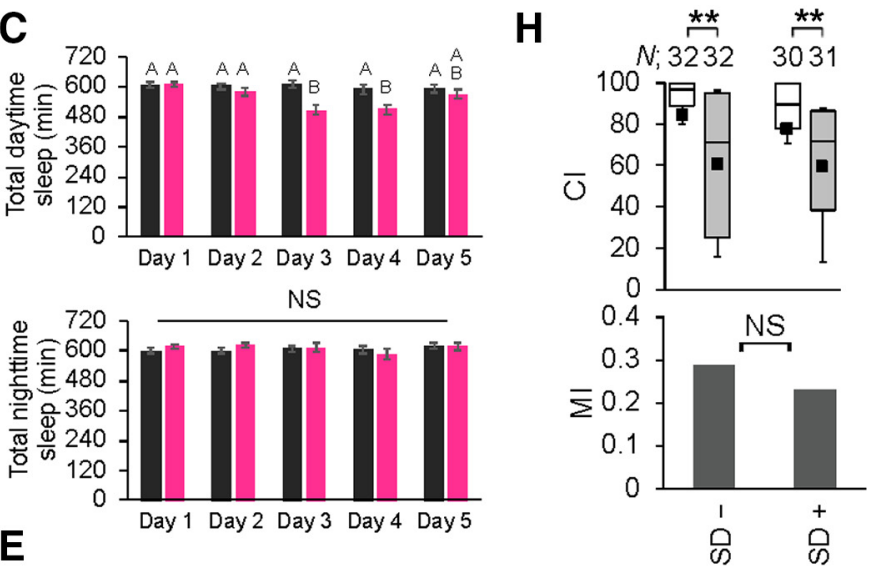

E

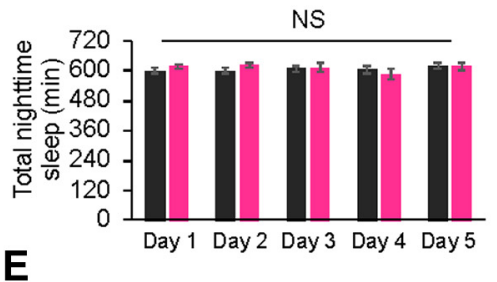

NS
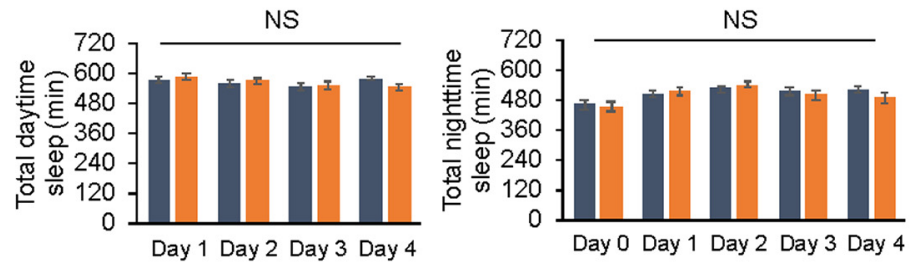

G
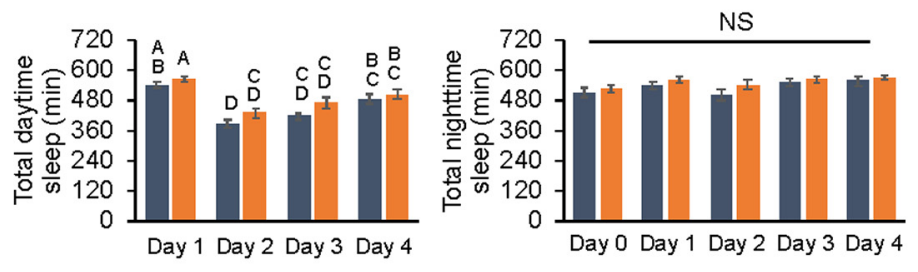

Figure 1. Light is essential for LTM maintenance. A, LTM was measured under various lighting conditions. Wild-type males were used in the experiments. Males were tested on Day 5 after $7 \mathrm{~h}$ conditioning. A schematic drawing of lighting conditions in courtship conditioning is shown on the left. The white box indicates day (light) and the black box indicates night (dark). The gray box indicates that experiments were conducted in darkness during the daytime. For Ml, asterisks indicate a comparison between the control (1) and test groups. (Figure legend continues.) 
also used. In $C r e b B-B$ experiments, we shifted PT to RT $\left(30^{\circ} \mathrm{C}\right)$ and vice versa during the two experimental phases: $10 \mathrm{~h}$ before the end of conditioning and $48-72 \mathrm{~h}$ after conditioning. To drive the expression of a UAS-Kir2.1 construct in Pdf neurons during the memory maintenance phase using the TARGET system, flies were kept at RT $\left(32^{\circ} \mathrm{C}\right)$ for $48-72$ $\mathrm{h}$ after conditioning. Subsequently, they were kept at PT.

Electrical silencing of large ventral lateral clock neurons. Pdf neurons form two clusters, small lateral ventral neurons (s-LNvs) and large lateral ventral neurons (1-LNvs). To assay whether 1 -LNv-specific electrical silencing affects LTM, two binary gene expression systems (GAL4/UAS and LexA/LexAop) combined with Flippase (FLP/FRT) were used. The specific target gene is expressed in GAL4- and LexA-coexpressing neurons using this system. R61G12-LexA and R14F03-GAL4 lines were used in the experiments.

Real-time quantitative reverse-transcription PCR. A PicoPure RNA Isolation Kit (KIT0204, ThermoFisher Scientific) was used for collecting total RNA from three whole brains in each genotype. cDNA was synthesized by the reverse transcription reaction using a QuantiTect Reverse Transcription Kit (205311, QIAGEN). Quantitative reverse-transcription PCR (qRTPCR) was performed using the THUNDERBIRD SYBR qPCR Mix (QPS201, TOYOBO) and a Chromo 4 detector (CFB-3240, MJ Research). The primer sequences (custom-made by Eurofins Genomics) used for qRT-PCR were as follows: Pdf-Forward, 5'-ATCGGGATCTCCTCGACTGG-3'; Pdf-Reverse, 5' -ATGGGCCCAAGGAGTTCTCG-3'; rp49-Forward, 5' AAGATCGTGAAGAAGCGCAC-3'; rp49-Reverse, 5' ${ }^{\prime}$-TGTGCACCAG GAACTTCTTG-3'. The expression level of each mRNA was normalized to that of $r p 49$ mRNA. The average of the normalized mRNA expression levels in control flies was calculated using data from five to six independent assays.

Luciferase assay. To test whether light or Pdfr activation promotes CrebB activity in MBs, the Luciferase (Luc) reporter was used (Tanenhaus et al., 2012). Luc should be expressed in MBs in the combination of CRE $>m$ Cherry::STOP $>l u c$ reporter, MB-GAL4, and UAS-FLP. Three MB-GAL4 lines (R41C10, R55D03, and R19B03) were used in these experiments. In vitro Luc activity was measured using a Luciferase Assay System (E1501, Promega). Three adult male heads were collected into a $1.5 \mathrm{ml}$ Eppendorf tube and homogenized in $50 \mu \mathrm{l}$ of Glo Lysis Buffer (E266A, Promega) at Zeitgeber time (ZT) 0-2. After centrifugation, 10 $\mu \mathrm{l}$ of the resulting supernatant and $50 \mu \mathrm{l}$ of Luciferin solution were used to analyze Luc activity. The luminescence of each sample was measured

\section{$\leftarrow$}

(Figure legend continued.) $\quad \boldsymbol{B}-\mathbf{G}$, Sleep amount was measured for $5 \mathrm{~d}$ using wild-type males. Data are presented as mean \pm SEM $\boldsymbol{B}$, Continuous sleep amounts of control (black line) and experimental flies (magenta line). In control flies, sleep amount was measured in LD. In experimental flies, sleep amount was measured for $2 \mathrm{~d}$ in $\mathrm{LD}$, which was subsequently shifted to $D D$ for $2 \mathrm{~d}$, and then back to LD. $N=31$, black line; $N=30$, magenta line. $C$, Total daytime and nighttime sleep amounts in control (black bars) and experimental flies (magenta bars). NS, Not significant. $N=31$, black bars; $N=30$, magenta bars. Nonparametric ANOVA (Kruskal-Wallis test) followed by post hoc analysis using the Steel-Dwass test was performed for multiple pairwise comparisons. Bars with the same letter indicate values that are not significantly different $(p>0.05)$. $\boldsymbol{D}$, Continuous sleep amounts in naive (dark blue line) and conditioned flies (orange line). $N=32$, dark blue line; $N=32$, orange line. $E$, Total daytime and nighttime sleep amounts in naive (dark blue bars) and conditioned flies (orange bars). Nonparametric ANOVA (Kruskal-Wallis test) was carried out. $N=32$ in each bar. $F$, Continuous sleep amounts in naive (dark blue line) and conditioned flies (orange line). $N=30$, dark blue line; $N=31$, orange line. Flies were deprived of sleep during the daytime on Days 2 and 3 after $7 \mathrm{~h}$ conditioning. SD, Sleep deprivation. G, Total daytime and nighttime sleep amounts in naive (dark blue bars) and conditioned flies (orange bars). Nonparametric ANOVA (Kruskal-Wallis test) followed by post hoc analysis using the Steel-Dwass test was performed for multiple pairwise comparisons. Bars with the same letter indicate values that are not significantly different $(p>0.05) . N=30$, dark blue bars; $N=31$, orange bars. $\boldsymbol{H}$, Memory on Day 5 was measured using sleep-deprived flies $(S D+)$. Control flies were kept in LD without sleep deprivation (SD - ). $\boldsymbol{A}, \boldsymbol{H}$, Box-and-whisker plots for a set of Cl data show 20th, 25th, 75th, and 80th centiles. In the box-and-whisker plots, the black square in each box indicates the mean, the line in each box is drawn at the median, white boxes indicate naive males, and gray boxes indicate conditioned males. The MannWhitney $U$ test was used for comparisons of $\mathrm{Cl}$. The permutation test with 10,000 random permutations was used for comparisons of Ml among experimental conditions. ${ }^{* *} p<0.01$, ${ }^{* * *} p<0.001$. NS, Not significant; $N$, Sample size in each box. using a luminometer (GloMax 20/20, Promega) and normalized to total protein concentration using a Protein Assay Kit (5000006, Bio-Rad). The UAS-FLP transgene used in this study displayed leaky expression of Luc [211-279 relative luminescence units (RLU)/mg]. Thus, first, in the control naive flies (UAS-FLP/CRE $>m$ Cherry::STOP $>l u c$ ), the mean normalized luminescence (mean $\mathrm{L}_{\text {control }}$ ) was calculated. CrebB activity index (CAI; RLU/mg) was defined as the difference between the "mean $\mathrm{L}_{\text {control }}$ " and the measured luminescence in each sample in each genotype, and finally, we calculated the mean CAI. To examine whether the induction of CrebB-B inhibits CrebB activity, we used UAS-FLP/hsCrebB-B; CRE $>m$ Cherry::STOP $>$ luc/R19B03 flies. For the heat-shock treatment, 3-4 d-old male flies were grouped into 20 flies per food vial. The flies were heat-shocked at $32^{\circ} \mathrm{C}$ for $3 \mathrm{~d}$. Luc activity was measured immediately after the heat-shock treatment at ZT $0-2$. To examine whether the activation of Pdf neurons increases CrebB activity in the MBs, we used UAS-FLP/R61G12-LexA; CRE > mCherry::STOP >luc LexAop-TrpA1/R41C10 flies. For the activation of Pdf neurons by TrpA1, the 3 - to 5 -d-old male flies were grouped into 20 flies per food vial. The flies were heat-shocked at $32^{\circ} \mathrm{C}$ for $2 \mathrm{~d}$ during $\mathrm{DD}$ (the second and third days after transfer). Luc activity was measured at ZT $0-2$ after the flies were returned to the normal LD cycle.

Immunohistochemistry. Immunohistochemistry was performed as previously described (Shimada et al., 2016). For Pdf staining, brains were stained with a mouse anti-Pdf antibody (PDF C7-s, Developmental Studies Hybridoma Bank at the University of Iowa; 1:200) followed by AlexaFluor 488 anti-mouse IgG or AlexaFluor 568 anti-mouse IgG (A11001 and A11004, ThermoFisher Scientific) as the secondary antibody (1: 1000). For GFP staining, brains were stained with a rabbit anti-GFP antibody (A11122, ThermoFisher Scientific; 1:200), followed by AlexaFluor 488 anti-rabbit IgG (A11008, ThermoFisher Scientific; 1:1000) as the secondary antibody. Fluorescence signals were observed under a confocal microscope [C2 (Nikon) or LSM710, (Zeiss)].

Quantitative analysis of Pdf immunoreactivity in l-LNvs. To examine whether temporal knockdown of $P d f$ in l-LNvs by the TARGET system inhibits Pdf immunoreactivity, Pdf-GAL4/UAS-Pdf RNAi; +/tubGAL $80^{\text {ts }}$ flies were used. Pdf-GAL4/+; +/tub-GAL80 ${ }^{\text {ts }}$ flies were used as the control. After eclosion, all flies were kept for 3-6 d at PT, and then the temperature was shifted to RT at ZT 8 . After $24 \mathrm{~h}$, the temperature was shifted again to PT. Subsequently, we dissected the brains for antibody staining $1 \mathrm{~h}$ after the temperature shift to PT (ZT 9). A confocal image stack of the brain hemisphere containing 1-LNvs was Z-projected into several sequential sections. $Z$-sections were collected at $1 \mu \mathrm{m}$ intervals. The signal intensity indicating Pdf immunoreactivity was quantified in a manually set region of interest of the cell body in each l-LNv using the NIS elements Ar (Nikon).

Sleep analysis. Single male flies (2-3 d old) were introduced into glass tubes ( $3 \mathrm{~mm}$ diameter $\times 75 \mathrm{~mm}$ length) containing fly food, and the glass tubes were set in a MB5 MultiBeam Activity Monitor (TriKinetics) to monitor the locomotor activity of individual flies. In this system, 17 independent infrared beams per glass tube were used to detect fly movement. When a fly repositions from one beam to the next, it was counted as one beam-crossing. Flies were acclimated in the glass tubes for $3 \mathrm{~d}$ in $\mathrm{LD}$ cycles at $25^{\circ} \mathrm{C}$ before measuring sleep amount. Locomotor activity data were collected at $1 \mathrm{~min}$ intervals for $5 \mathrm{~d}$ and analyzed with a Microsoft Excel-based program as previously described (Kume et al., 2005). Sleep was defined as behavioral inactivity for 5 min or more (Huber et al., 2004). Total sleep amount during the day or night was analyzed as previously described (Shimada et al., 2016).

To deprive flies of sleep, a MB5 MultiBeam Activity Monitor with glass tubes each containing one naive or conditioned fly was horizontally shaken using a shaker (NJ-022NS, Nissin). The shaker was placed inside a temperature-regulated incubator (MIR-254, Sanyo Electric). The shaking speed was set to $200 \mathrm{rpm}$. On Days 2 and 3 after $7 \mathrm{~h}$ conditioning, the MB5 MultiBeam Activity Monitor was shaken for $20 \mathrm{~s}$ per 3 min during only the daytime. All experiments were performed in $12 \mathrm{~h} \mathrm{LD}$ cycles at $25.0 \pm 0.5^{\circ} \mathrm{C}$.

Experimental design and statistical analyses. All the statistical analyses were performed using IBM SPSS Statistics 22 or BellCurve for Excel (Social Survey Research Information) except for the comparisons of MI. 
A
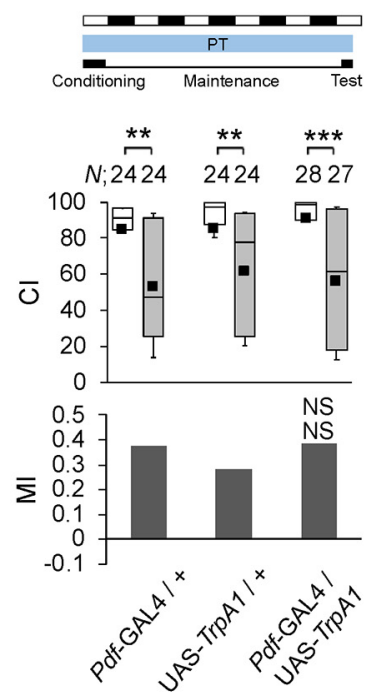

E
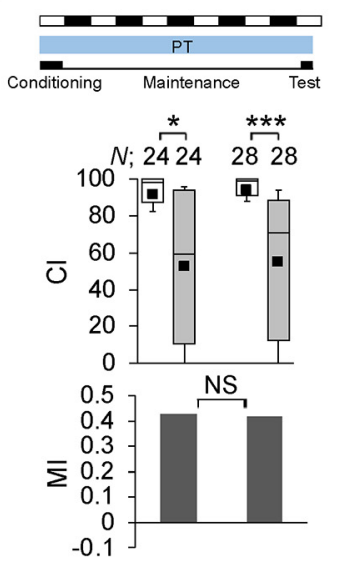

UAS-Kir2.1 $+\quad+$ tub-GAL80ts $+\quad+$ Pdf-GAL4 +
B
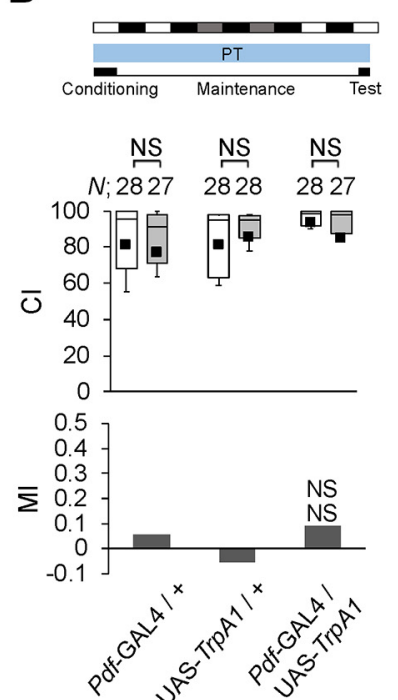

F

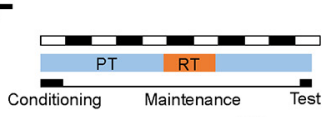

*** NS
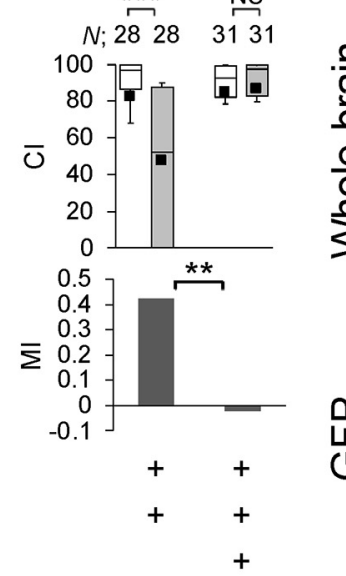

G
C
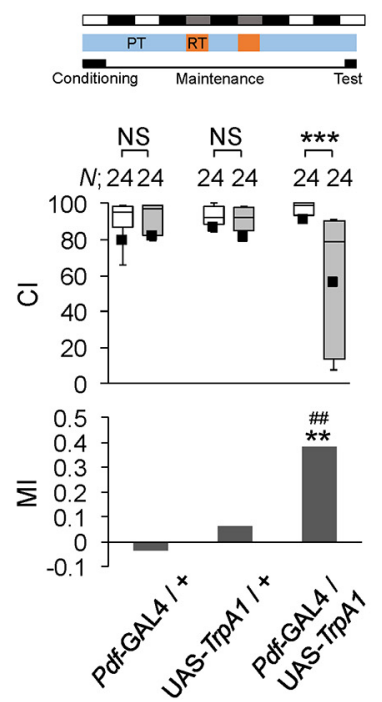

H
D
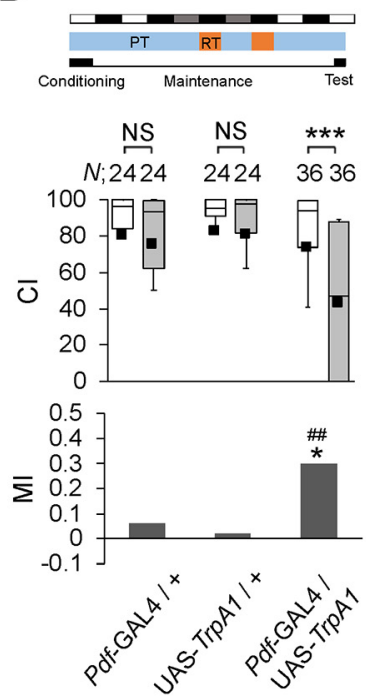

I
R61G12-LexA
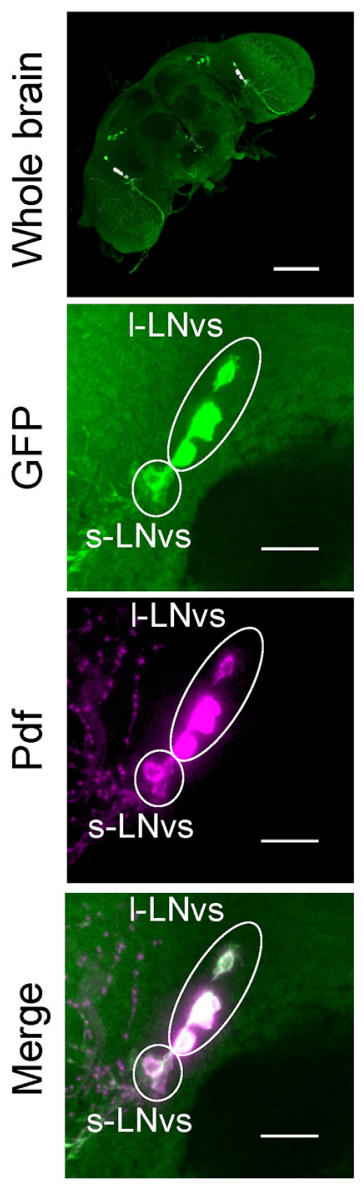

R14F03
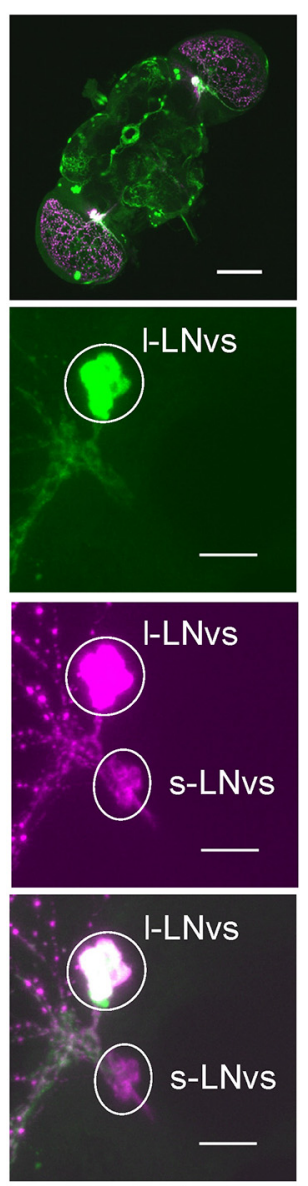

R61G12-LexA $\cap R 14 F 03$
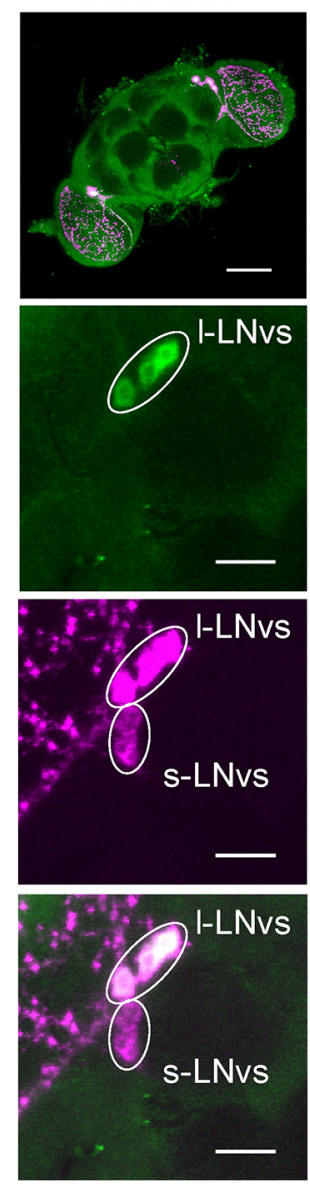

Figure 2. Activity of Pdf neurons is required for LTM maintenance. A-D, Temporal activation of Pdf neurons compensates for DD-dependent LTM impairment. Pdf-GAL4/+, UAS-TrpA1/+, and Pdf-GAL4/UAS-TrpA1 males were used. For MI, asterisks indicate a comparison between $\mathrm{F}_{1}$ (GAL4/UAS) and GAL 4 control flies, and hash marks indicate a statistical comparison between $\mathrm{F}_{1}$ and UAS control flies. $\boldsymbol{A}$, All experiments were performed at PT. $\boldsymbol{B}, 0 \mathrm{n}$ Days 2 and 3 after $7 \mathrm{~h}$ conditioning, flies were kept in DD. $\boldsymbol{C}, 0 \mathrm{n}$ Days 2 and 3 after $7 \mathrm{~h}$ conditioning, flies were kept at $34^{\circ} \mathrm{C}$ during the period between CT 0 and CT 8. D, On Days 2 and 3 after $7 \mathrm{~h}$ conditioning, flies were kept at $34^{\circ} \mathrm{C}$ during the period between CT 12 and CT 20. E, F, UAS-Kir2.1/Pdf-GAL4; tub-GAL80 ${ }^{\text {ts }} /+$ flies were used. UAS-Kir2.1/tub-GAL80 ${ }^{\text {ts }}$ flies were used as the control. $\boldsymbol{E}$, All experiments were performed at PT $\left(25^{\circ} \mathrm{C}\right)$. $\boldsymbol{F}$, Flies were kept at RT $\left(32^{\circ} \mathrm{C}\right)$ for $48-72 \mathrm{~h}$ after $7 \mathrm{~h}$ conditioning. $\mathbf{G}-\boldsymbol{I}$, Confocal section images of whole brain and Pdf neurons. R61G12-LexA/LexAop2-mCD8::GFP (G), R14F03/UAS-mCD8::GFP (H), and R61G12-LexA/LexAop-FLPL; (Figure legend continues.) 
In all statistical analyses except for the comparisons of MI, the Kolmogorov-Smirnov test was used to determine whether the data are normally distributed. In the statistical analysis of CI, when the data were not distributed normally, we performed the log transformation of the data. When the basic data or transformed data were normally distributed, Student's $t$ test was used for comparisons. When the basic data and transformed data were not distributed normally, we used the MannWhitney $U$ test for comparisons. In the statistical analysis of MI, the permutation test with 10,000 random permutations was used $\left(H_{0}\right.$, the difference between experimental and control groups is 0 ). The free statistical package $\mathrm{R}$ was used for these tests (Koemans et al., 2017). In qRT-PCR, the mean $( \pm$ SEM) ratio was calculated using data from four to six independent assays. Because the log-transformed data were normally distributed, one-way ANOVA followed by post hoc analysis using Scheffe's test was used. In the Luciferase assay, when the basic data were distributed normally, Student's $t$ test was used for comparisons of two means, and one-way ANOVA followed by post hoc analysis using Scheffe's test was performed for multiple comparisons. When the basic data or log-transformed data were not normally distributed, we performed nonparametric ANOVA (Kruskal-Wallis test) followed by the Steel-Dwass test for multiple comparisons. In quantitative analysis of Pdf immunoreactivity, all image data were acquired under identical conditions. When the basic data or log-transformed data were distributed normally, Student's $t$ test was used. When they were not normally distributed, we performed the Mann-Whitney $U$ test. In sleep analysis, because the basic data were distributed normally, Student's $t$ test was performed to determine the significance of the difference between two means. For multiple comparisons, since the basic data or log-transformed data were not normally distributed, we performed nonparametric ANOVA (Kruskal-Wallis test) followed by the Steel-Dwass test for multiple comparisons.

\section{Results}

\section{Light is essential for LTM maintenance}

To determine whether lighting conditions affect LTM in Drosophila, the courtship conditioning assay was performed (Siegel and Hall, 1979; Sakai et al., 2004; Griffith and Ejima, 2009; Keleman et al., 2012). In this assay, males receive stressors from nonreceptive mated females (e.g., sexual rejection) to block successful mating (conditioning; S. S. Lee et al., 2017), and memory is subsequently observed as experience-dependent courtship suppression toward virgin females. One hour conditioning generates short-term memory (STM), which persists for at least $8 \mathrm{~h}$, whereas $7 \mathrm{~h}$ conditioning induces LTM, which persists for at least $5 \mathrm{~d}$ (Sakai et al., 2004, 2012; Ishimoto et al., 2009). The courtship activity of naive and conditioned males was quantified using CI; subsequently, MI was calculated to quantify courtship memory (see Materials and Methods). When males were conditioned for $7 \mathrm{~h}$ in light or darkness and the conditioned males were subsequently kept under LD cycles until the test, they showed lower courtship activity on Day 5 (i.e., $5 \mathrm{~d}$ after conditioning) than naive males, and there was no significant difference in MI between these flies [Fig. $1 A$; (1) vs (2), permutation test; $p=$ 0.5558 ], indicating that conditioning in darkness has no adverse effects on LTM. However, when flies were conditioned in light

(Figure legend continued.) UAS $>$ STOP $>$ Kir2.1::eGFP/R14F03 flies (I) were used. Scale bars: 50 or $10 \mu \mathrm{m}$. Green, GFP; magenta, Pdf. J, I-LNv-specific silencing impairs LTM. R61G12-LexA/ LexAop-FLPL;UAS $>$ STOP $>$ Kir2.1/R14F03 flies were used. For MI, asterisks indicate a comparison between I-LNv-silenced flies and the LexA/LexAop control (R61G12-LexA/LexAop-FLPL), and the hash mark indicates a comparison between I-LNv-silenced flies and the GAL4/UAS control (UAS $>$ STOP $>$ Kir2.1/R14F03). The permutation test was used (LexA/LexAop control vs I-LNv-silenced flies, $p=0.0011 ;$ GAL4/UAS control vs I-LNv-silenced flies, $p=0.0113) . \boldsymbol{A}-\boldsymbol{F}, \boldsymbol{J}$, The Mann-Whitney $U$ test was used for comparisons of $\mathrm{Cl}$. The permutation test with 10,000 random permutations was used for comparisons of MI. ${ }^{*} p<0.05,{ }^{* *} p<0.01$, ${ }^{* * *} p<0.001$, $\# p<0.05$, \#\#p <0.01. NS, Not significant; $N$, sample size in each box. and then kept in DD after the conditioning and before the test, LTM was severely impaired [Fig. $1 A$; (1) vs (3), permutation test, $p=0.0020]$. DD for $2 \mathrm{~d}$ after conditioning was sufficient to impair LTM [Fig. 1A; (1) vs (6), permutation test; $p=0.0020$ ], but not DD for only $1 \mathrm{~d}$ [ Fig. $1 A$; (1) vs (4), permutation test, $p=$ 0.8290 ; (1) vs (5), permutation test, $p=0.8902$ ], indicating that flies cannot maintain their LTM when DD lasts $>2 \mathrm{~d}$. In constant light (LL), the Drosophila circadian clock does not work normally, and flies show arrhythmic locomotor activity (Qiu and Hardin, 1996). When flies were kept in LL after conditioning, their LTM was intact [Fig. $1 A$; (1) vs (7), permutation test, $p=$ 0.2110 ], as previously reported (Sakai et al., 2004). Furthermore, the LTM of several clock mutants except for period (per) mutants is intact (Sakai et al., 2004). Thus, light input, but not the circadian clock, is necessary for LTM maintenance.

Sleep plays an important role in the consolidation of Drosophila courtship memory (Ganguly-Fitzgerald et al., 2006; Donlea et al., 2011). However, it remains unclear whether an abnormal sleep phenotype causes the disturbed LTM maintenance of flies kept in DD. To examine whether light conditions affect Drosophila sleep (see Materials and Methods), sleep amount was measured in flies kept in LD and DD (Fig. 1B). The mean amount of daytime sleep of flies kept in DD was lower than that of flies kept in LD (Fig. $1 C$; total daytime sleep: Kruskal-Wallis test, $H_{(9)}=$ 75.113, $p<0.0001$; total nighttime sleep: Kruskal-Wallis test, $\left.H_{(9)}=7.534, p=0.3570\right)$. Next, using naive and conditioned males, we measured sleep amount in LD after courtship conditioning (Fig. 1D,E). As previously reported (Ganguly-Fitzgerald et al., 2006), the amount of daytime sleep during the period between the termination of conditioning (ZT8) and the lights off (ZT12) was higher in conditioned males than in naive males (Student's $t$ test, $\left.t_{(62)}=-3.7754, p=0.0003\right)$. However, from ZT 12 on Day 0, no significant difference between naive and conditioned males was detected in the amount of daytime sleep (Fig. $1 E$; Kruskal-Wallis test, $\left.H_{(7)}=6.829, p=0.447\right)$ or nighttime sleep ( $1 E$; Kruskal-Wallis test, $H_{(9)}=14.891, p=0.094$ ). When flies kept in LD were slightly deprived of sleep to adjust the amount of daytime sleep to the level of that in DD (Fig. 1F, G; total daytime sleep: Kruskal-Wallis test, $H_{(7)}=75.113, p<$ 0.0001; total nighttime sleep: Kruskal-Wallis test, $H_{(7)}=7.534$, $p=0.3570)$, LTM in flies with slight sleep deprivation was not attenuated (Fig. $1 H$; SD - vs SD + , permutation test, $p=0.7204$ ). Thus, LTM impairment induced by DD does not simply result from the reduced amount of sleep.

\section{Activity of Pdf neurons regulates light-dependent LTM maintenance}

Because light activates Pdf neurons, it is possible that the activation of Pdf neurons restores LTM in DD. Thus, we examined whether the temporal activation of Pdf neurons induced by the temperature-sensitive cation channel TrpAl can compensate for the DD-dependent LTM impairment (Fig. 2A-D). When Pdf neurons were activated in $P d f$-GAL4/UAS-TrpA1 flies on each subjective day or night in DD for $2 \mathrm{~d}$ after conditioning, LTM was maintained for $5 \mathrm{~d}$ in $P d f$-GAL4/UAS-TrpA1 flies (Fig. 2C,D; permutation test; $C$, UAS control vs $\mathrm{F}_{1}, p=0.0090$, GAL4 control vs $\mathrm{F}_{1}, p=0.0038 ; D$, UAS control vs $\mathrm{F}_{1}, p=0.0074$, GAL4 control vs $\left.\mathrm{F}_{1}, p=0.0122\right)$. Under the same temperature-shift conditions, GAL4 and UAS control flies still showed LTM impairment (Fig. $2 C, D)$. Thus, this finding indicates that the activation of Pdf neurons during either a subjective day or night is sufficient to restore LTM. Consistently, the electrical silencing of Pdf neurons during the memory maintenance phase attenuated LTM in LD 
A
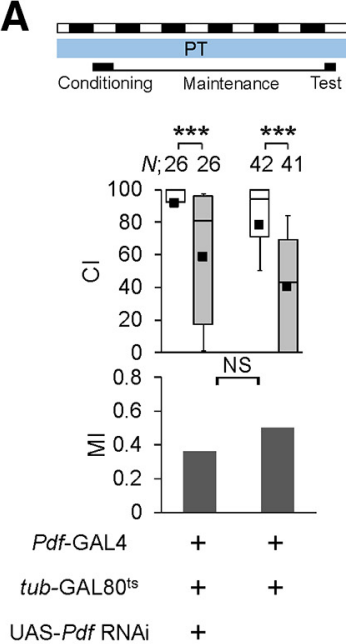

E

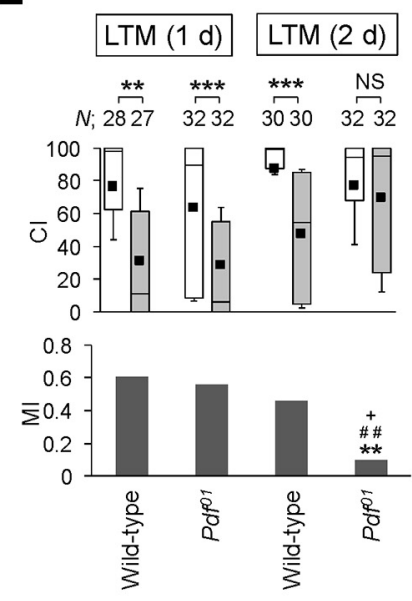

B
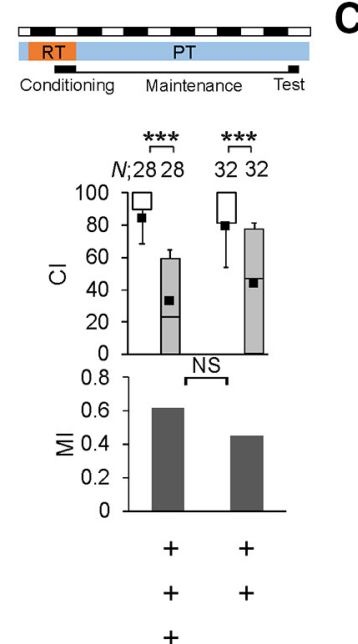

$\mathbf{F}$
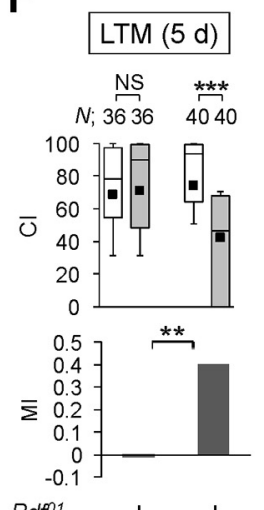

Pdfo1 $+\quad+$

\section{$c$}

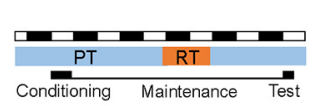

D
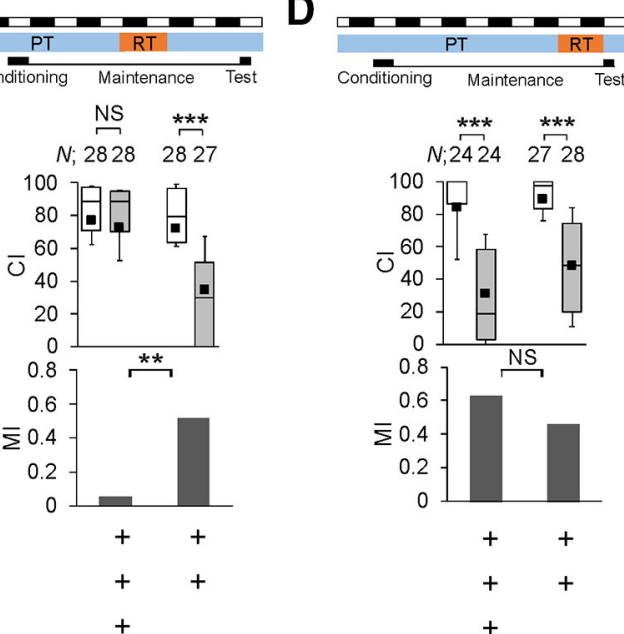

G
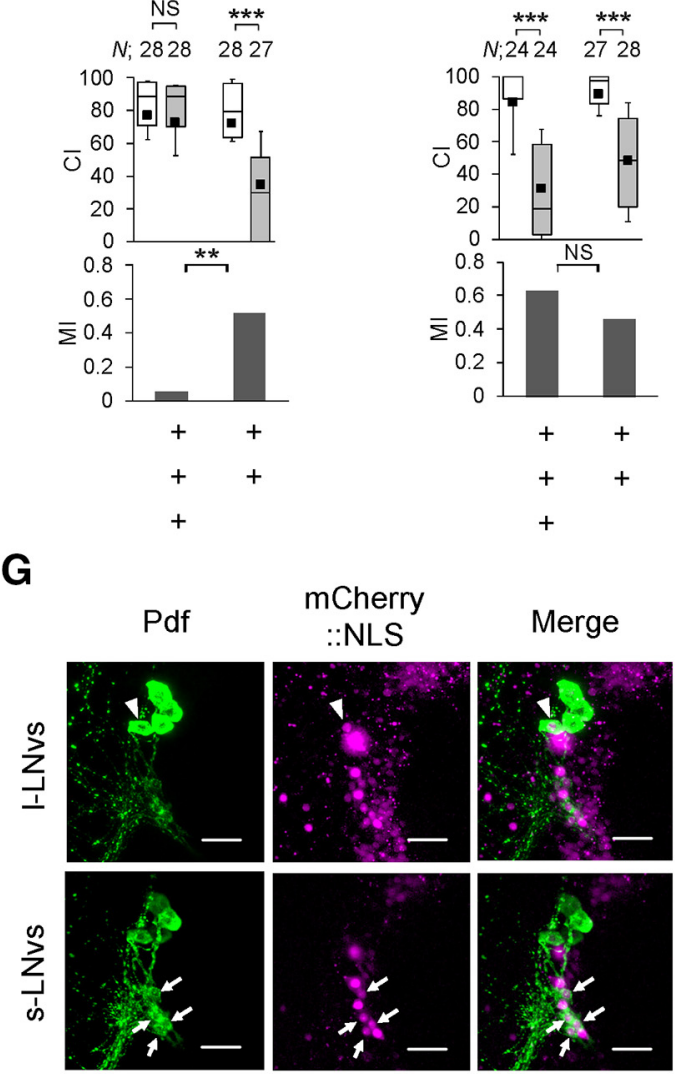

Merge
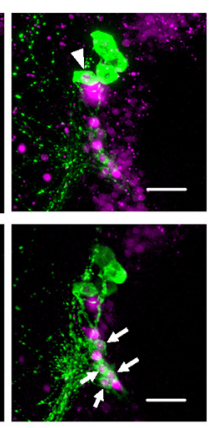

H

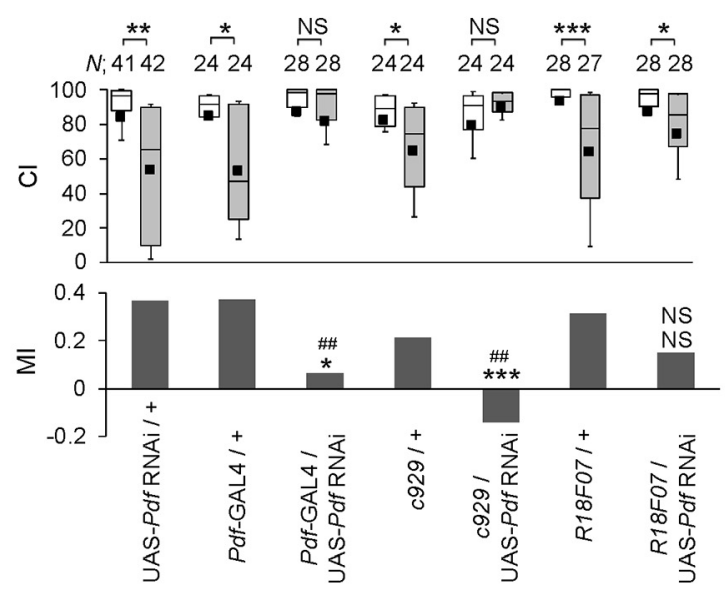

Figure 3. Pdf expression is essential for LTM maintenance. $\boldsymbol{A}-\boldsymbol{F}, \boldsymbol{H}$, Males were tested on Days 1,2 , or 5 after $7 \mathrm{~h}$ conditioning. In the box-and-whisker plots, the white boxes indicate naive males and the gray boxes indicate conditioned males. The Mann-Whitney $U$ test was used for comparisons of Cl. The permutation test with 10000 random permutations was used for comparisons of $M I$. ${ }^{*} p<0.05,{ }^{* *} p<0.01,{ }^{* * *} p<0.001$, \#\#p $<0.01$. NS, Not significant; $N$, sample size in each box. A, All experiments were performed at PT ( $\left.25^{\circ} \mathrm{C}\right)$. B, Flies were kept at RT for $24 \mathrm{~h}$ before the end

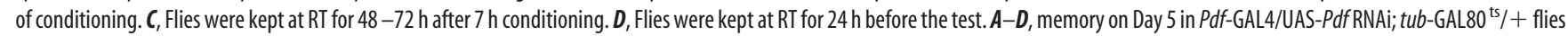

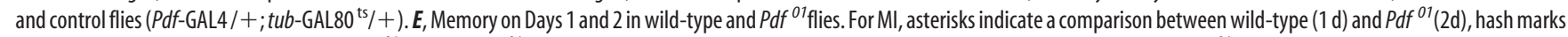

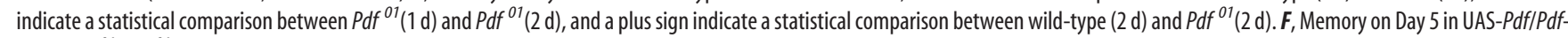
GAL4; $P d f^{01} / P d f{ }^{01}$ flies and control flies. G, Confocal section images at Pdf neuron level of the adult brain. Triangles, I-LNvs; arrows, s-LNvs. Scale bars, $50 \mu \mathrm{m}$. UAS-mCherry::NLS/R18F07 flies were used. Magenta, mCherry::NLS; green, Pdf. $\boldsymbol{H}$, Knockdown of Pdf in I-LNvs and/or s-LNvs using three GAL4 drivers (Pdf-GAL4, C929, and R18F07). For MI, asterisks indicate a comparison between $\mathrm{F}_{1}$ and GAL4 control flies, and hash marks indicate a statistical comparison between $\mathrm{F}_{1}$ and UAS control flies. The permutation test was used (UAS control vs $C 929 /$ UAS-Pdf RNAi, $p=0.0028 ;$ GAL4 control vs (929/UAS-Pdf RNAi, $p<0.0001$; UAS control vs R18F07/UAS-Pdf RNAi, $p=0.0588$; GAL4 control vs R18F07/UAS-Pdf RNAi, $p=0.1536$ ). 
A

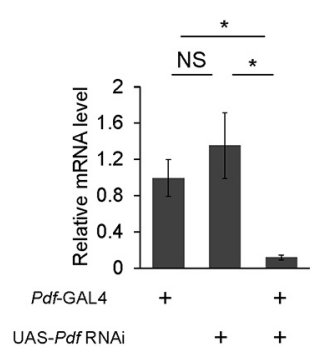

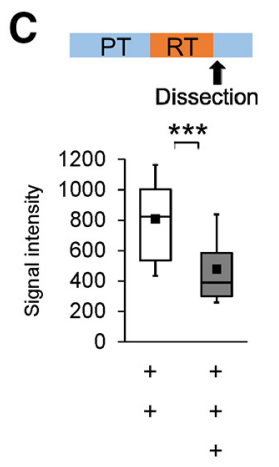

Figure 4. Real-time qRT-PCR analysis and quantitative analysis of Pdf immunoreactivity. $A$, Analysis of Pdf mRNA expression level. Pdf-GAL4 was used for the induction of Pdf RNAi. Mean \pm SEM was calculated from five to six replicates. One-way ANOVA followed by post hoc analysis using Scheffe's test for multiple pairwise comparisons was used. ${ }^{*} p<0.05$. NS, Not significant. $B, C$, Quantitative analysis of Pdf immunoreactivity in I-LNvs. Adult brains were dissected at ZT 9 . In the box-and-whisker plots, white boxes indicate Pdf-GAL4/+; tubGAL80 ${ }^{\text {ts }} /+$ flies and gray boxes indicate Pdf-GAL4/UAS-Pdf RNAi; tub-GAL 80 ts $/+$ flies. $B$, All experiments were performed at $\mathrm{PT}\left(25^{\circ} \mathrm{C}\right) . N=26$, white box; $N=27$, gray box. Student's $t$ test was used for statistical analysis. C, Flies were kept at $\mathrm{RT}\left(30^{\circ} \mathrm{C}\right)$ for $24 \mathrm{~h} . N=50$, white box; $N=$ 38 , gray box. The Mann-Whitney $U$ test was used for statistical analysis. ${ }^{* * *} p<0.001$.

(Fig. 2E, F; permutation test: $E, p=0.9492 ; F, p=0.0014$ ). Together, these results indicate that the activity of Pdf neurons regulates light-dependent LTM maintenance.

\section{Pdf expression is critical for LTM maintenance in LD}

We next determined the temporal requirement of Pdf for LTM maintenance. For this purpose, we performed temporal knockdown of $P d f$ in Pdf neurons using the TARGET system (McGuire et al., 2003) and RNA interference (RNAi) technology. To demonstrate that the Pdf signaling pathway regulates light-driven LTM maintenance, we temporally knocked down $P d f$ in Pdf neurons (Fig. 3A-D). The effectiveness of $P d f$ RNAi was confirmed by qRT-PCR (Fig. $4 A$; one-way ANOVA, $F_{(2,12)}=6.954, p=$ 0.0099; Scheffe's multiple comparisons, GAL4 control vs $\mathrm{F}_{1}, p=$ 0.0193 , UAS control vs $\left.F_{1}, p=0.0291\right)$ and immunostaining using an anti-Pdf antibody (Fig. $4 B, C$; Student's $t$ test in $B, t_{(49)}=$ -1.801, $p=0.0778$; Mann-Whitney $U$ test in $C, U=365, p<$ 0.0001). To knockdown Pdf during the memory consolidation, maintenance or test phase, the temperature was raised to $30^{\circ} \mathrm{C}$ for $24 \mathrm{~h}$ during the three experimental periods (Fig. $3 B-D$ ): starting at $24 \mathrm{~h}$ before the end of conditioning, $48-72 \mathrm{~h}$ after conditioning (memory maintenance phase), and $24 \mathrm{~h}$ before the test initiation. When flies were kept for $24 \mathrm{~h}$ before the test initiation at RT, Pdf should remain suppressed during the 10 min test. LTM was impaired only when $P d f$ was knocked down during the memory maintenance phase (Fig. $3 A-D$; permutation test: $A, p=0.3274$; $B, p=0.1904 ; C, p=0.0034 ; D, p=0.1290)$. Although memory on Day 1 remained intact in $P d f^{01}$-null mutant flies after $7 \mathrm{~h}$ conditioning (Fig. 3E; wild-type-1d vs $P d f^{01}-1 \mathrm{~d}$; permutation test, $p=0.9646), P d f^{01}$ mutant flies showed memory impairment on Day 2 (Fig. $3 E$; wild-type-1d vs $P d f^{O 1}-2 \mathrm{~d}$; permutation test, $p=0.0020)$, suggesting that memory consolidation completes within $1 \mathrm{~d}$ after conditioning and LTM has already entered the maintenance phase on Day 2 after conditioning. Thus, Pdf seems to be dispensable for memory consolidation. In addition, $P d f^{01}$ mutant flies showed memory impairment on Day 5, which was rescued by $P d f$ expression (Fig. $3 F$; permutation test, $p=0.0020$ ). This finding also supports the idea that $\mathrm{Pdf}$ is required for maintaining LTM for $>1 \mathrm{~d}$.
We next used two additional GAL4 drivers, c929 and R18F07, to further investigate neuronal cell types involved in Pdfmediated LTM maintenance. $c 929$ drives the expression of GAL4 in peptidergic neurons including l-LNvs but not s-LNvs (Taghert et al., 2001; Shimada et al., 2016), and R18F07 drives GAL4 expression in all s-LNvs and only weakly in one of the l-LNvs (Fig. 3G). Pdf knockdown in c929-positive neurons impaired LTM, but not that in $\mathrm{R} 18 \mathrm{~F} 07$-positive neurons (Fig. $3 \mathrm{H}$; the probability in each permutation test is shown in the figure legend). In addition, we examined whether l-LNv-specific electrical silencing impairs LTM. First, we confirmed that LexA is expressed in 1-LNvs and s-LNvs in R61G12-LexA (Fig. 2G) and GAL4 is expressed in only l-LNvs in R14F03-GAL4 (Fig. $2 H$ ). Next, we confirmed that Kir2.1::eGFP is expressed in all 1-LNvs but not in s-LNvs in R61G12-LexA/LexAop-FLPL; UAS >STOP > Kir2.1::eGFP/R14F03 flies (Fig. 2I). Moreover, l-LNv-specific electrical silencing also impaired LTM (Fig. 2J; the probability in each permutation test is shown in the figure legend). Together, it is most likely that Pdf expression in 1-LNvs is essential for LTM maintenance.

\section{Pdfr is essential for light-dependent CrebB activity in MB $\boldsymbol{\alpha} / \boldsymbol{\beta}$ neurons}

A Drosophila homolog of CREB (CrebB) is required for the consolidation and maintenance of olfactory memory (Yin and Tully, 1996; Hirano et al., 2016). Our previous studies demonstrated that the consolidation of courtship memory is also regulated by CrebB (Sakai et al., 2004; Ishimoto et al., 2009). Thus, using a repressor isoform of CrebB (CrebB-B; also known as dCREB2-b), we first examined whether CrebB is also required for the maintenance of courtship memory (Fig. $5 A-C$ ). LTM was attenuated when CrebB-B was expressed in $\mathrm{MB} \alpha / \beta$ neurons (Fig. $5 D$ ) during conditioning (Fig. $5 B$; permutation test, $p<0.0001$ ) or the memory maintenance phase (Fig. $5 C$; permutation test, $p=$ 0.0054 ), indicating that $\mathrm{CrebB}$ in $\mathrm{MB} \alpha / \beta$ neurons is required for both the consolidation and maintenance of LTM. On the other hand, CrebB in $\mathrm{MB} \gamma$ neurons (Fig. $5 E$ ) during conditioning, but not during the maintenance phase, attenuated LTM (Fig. $5 B, C$; permutation test in $B, p=0.0022$; permutation test in $C, p=$ 0.37 ), suggesting that CrebB in $\mathrm{MB} \gamma$ neurons is required only for memory consolidation. Unlike the $\mathrm{MB} \alpha / \beta$ and $\gamma$ neurons, $\mathrm{MB}$ $\alpha^{\prime} / \beta^{\prime}$ neurons (Fig. $5 F$ ) had no effect on CrebB-dependent LTM (Fig. $5 A-C$; permutation test in $B, p=0.2958$; permutation test in $C, p=0.5666)$.

We next examined whether light activates CrebB transcription in MBs. To estimate CrebB activity, we used the CRE>mCherry:: $\mathrm{STOP}>$ luciferase $(l u c)$ reporter, which is a luc-based reporter gene under the control of CrebB-binding sites (CRE; Tanenhaus et al., 2012). Using this reporter construct, in which the $m$ Cherry::STOP sequence is flipped out in the presence of the GAL4-induced recombinase FLP, we can measure the MB-specific transcriptional activity of CrebB with MB-GAL4. The induction of a CrebB repressor driven by a heat-shock promoter severely attenuated the CRE-dependent Luc activity (Fig. 6A; Kruskal-Wallis test, $H_{(3)}=24.6833, p<$ 0.0001 ), when the pan-neuronal MB line R19B03-GAL4 was used (Pan and Baker, 2014). Thus, this reporter can reliably be used as an indicator of CrebB activity. When naive flies were kept in $\mathrm{LD}$, robust CrebB activity was detected in $\mathrm{MB} \alpha / \beta$ neurons, but not in $\mathrm{MB} \gamma$ neurons [Fig. $6 B,(1)$ and (2); statistic values are shown in the figure legend]. When they were kept in DD for $1 \mathrm{~d}$, the CrebB activity in $\mathrm{MB} \alpha / \beta$ neurons decreased by $\sim 50 \%$ compared with that in control flies [Fig. $6 B,(3)$ ]. On Day 2 of DD, it decreased further [Fig. $6 B,(4)$ ], indicating that the CrebB activity in $\mathrm{MB} \alpha / \beta$ neurons is lightdependent. Furthermore, we examined whether the Pdf receptor 
A
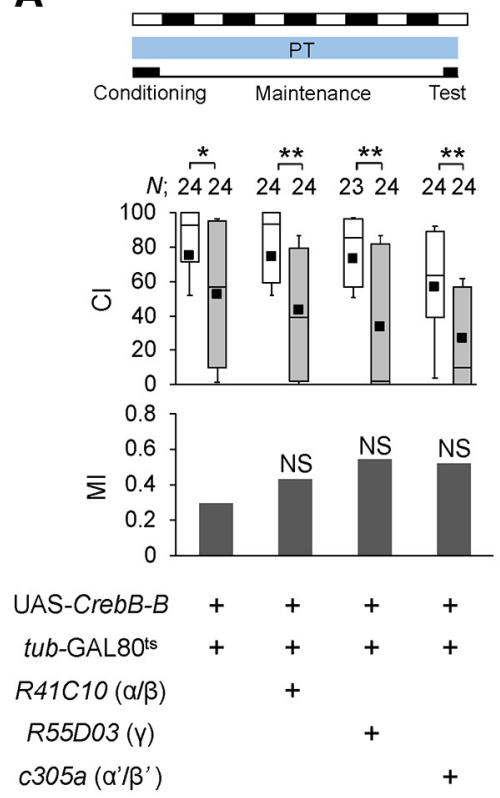

D

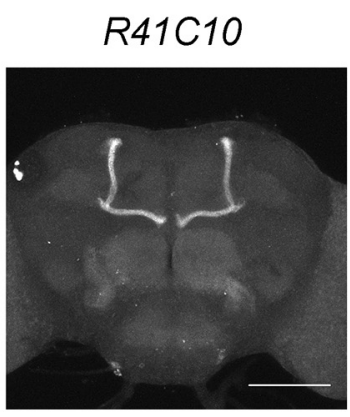

B
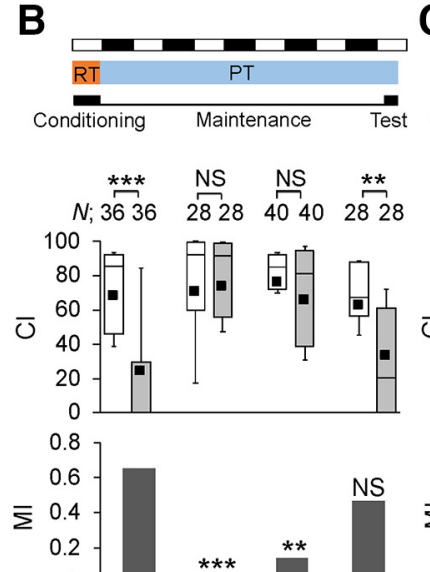

0

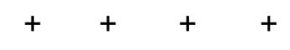

+
+

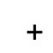

E

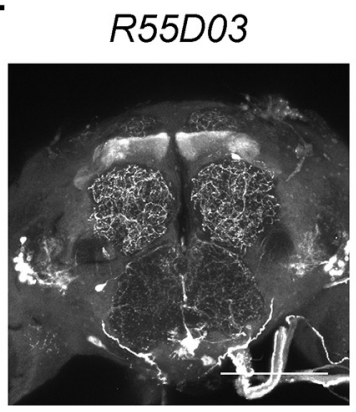

C
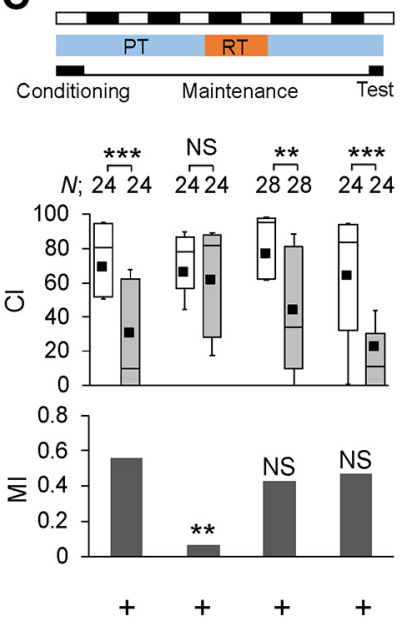

$+\quad+\quad+$

$+$

$+$

F

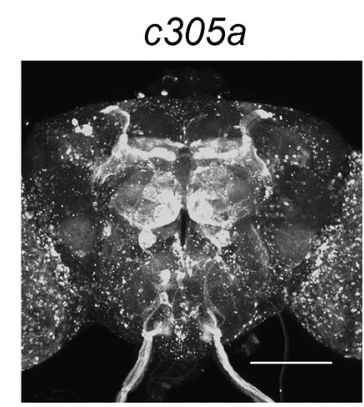

Figure 5. Temporal expression of CrebB repressor in MB neurons. A-C, The Mann-Whitney $U$ test was used for comparisons of $\mathrm{Cl}$. The permutation test with 10,000 random permutations was used for comparisons of MI. For MI, asterisks indicate a comparison between the UAS control and test groups. ${ }^{*} p<0.05,{ }^{* *} p<0.01,{ }^{* * *} p<0.001$. NS, not significant; $N$, sample size in each box. All flies were raised at $25^{\circ} \mathrm{C}$. The temperature was shifted during two experimental periods. A, All experiments were performed at $\mathrm{PT}\left(25^{\circ} \mathrm{C}\right)$. $\boldsymbol{B}$, Flies were kept at $\mathrm{RT}\left(30^{\circ} \mathrm{C}\right)$ for $10 \mathrm{~h}$ before the end of conditioning. C, Flies were kept at RT for $48-72 \mathrm{~h}$ after $7 \mathrm{~h}$ conditioning. D-F, Stacked confocal images of the adult brain. R41C10/UAS-mCD8::GFP (D), R55D03/UAS-mCD8::GFP (E), and c305a/UAS-mCD8::RFP (F) flies were used. Scale bars, $100 \mu \mathrm{m}$.

(Pdfr) regulates the light-dependent CrebB activity. As was observed in $P d f^{01}$ flies, $P d f r$-null mutant $\left(P d f r^{5304}\right)$ flies also showed severe LTM impairment [Fig. $6 C$; permutation test, $p=0.0034$ ]. In LD, CrebB activity was severely attenuated in $\mathrm{MB} \alpha / \beta$ neurons with the $P d f r$-null mutant background [Fig. $6 B,(1)$ vs (5)]. Furthermore, in $\mathrm{DD}$, the activation of Pdf neurons by TrpA1 increased the CrebB activity in $\mathrm{MB} \alpha / \beta$ neurons (Fig. $6 D$; Mann-Whitney $U$ test, $U=35$, $p=0.0043)$. Thus, the Pdf/Pdfr signaling pathway is essential for light-driven CrebB activity in $\mathrm{MB} \alpha / \beta$ neurons.

Next, the effect of $7 \mathrm{~h}$ conditioning on CrebB activity was examined. The CrebB activity in $\mathrm{MB} \alpha / \beta$ and $\gamma$ neurons increased immediately after $7 \mathrm{~h}$ conditioning (Fig. $6 E ; \alpha / \beta$ neurons: Student's $t$ test, $t_{(16)}=-2.9747, p=0.0089 ; \gamma$ neurons: MannWhitney $U$ test, $U=72, p=0.028$ ). This finding is consistent with a previous report (Ishimoto et al., 2009). However, no conditioning-dependent increase in CrebB activity was observed during the day on Days 1 and 2 or during the night on Day 2 after $7 \mathrm{~h}$ conditioning (Fig. $6 F-H ; F, \alpha / \beta$ neurons: Student's $t$ test, $t_{(12)}=$ $-0.3655, p=0.7211, \gamma$ neurons: Student's $t$ test, $t_{(14)}=-0.5828$, $p=0.5693 ; G, \alpha / \beta$ neurons: Student's $t$ test, $t_{(16)}=1.1219, p=$ $0.2749 ; H, \alpha / \beta$ neurons: Student's $t$ test, $t_{(16)}=-0.2441, p=$ $0.8102)$.

\section{Discussion}

In nocturnal mice, an ultradian LD cycle condition induces spatial learning defects, although such an aberrant light condition does not impair the molecular clock or sleep amount (LeGates et al., 2012). The ultradian LD cycle also attenuates hippocampal LTP (Fernandez et al., 2018). In contrast, a short pulse of white light during the night before learning enhances long-lasting fear memory through the activation of hippocampal p21-activated kinase 1 (Shan et al., 2015). In humans, who are naturally diurnal, prior exposure to orange light promotes working memory (Chellappa et al., 2014). Thus, regardless of the nocturnal or diurnal nature of animals, lighting conditions can positively or negatively modify the acquisition or consolidation of memories (Cajochen et al., 2011; LeGates et al., 2012; Chellappa et al., 2014; Shan et al., 2015). However, little is known on whether environmental light affects LTM maintenance. In this study using Drosophila, we demonstrated for the first time that environmental light is an essential factor for the appropriate maintenance of LTM.

The Drosophila Pdf neuropeptide regulates various biological phenomena such as circadian behavioral rhythms, light-driven 
A

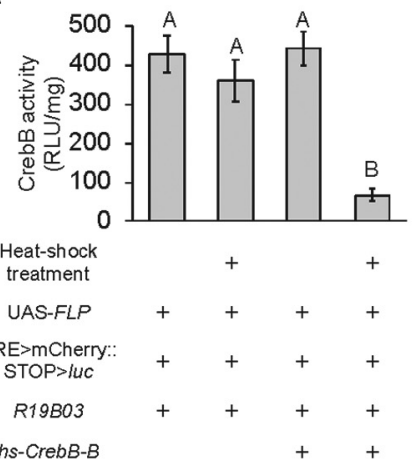

$\mathbf{E}$
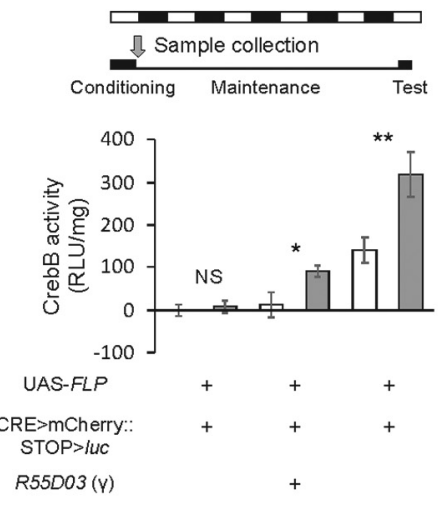

B

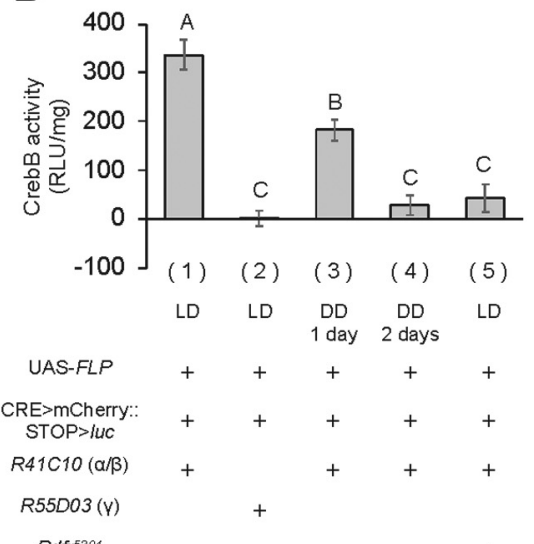

$\mathbf{F}$
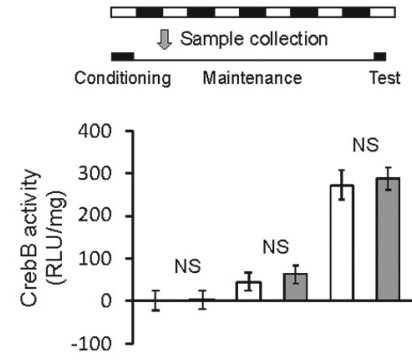

$\begin{array}{lll}+ & + & + \\ + & + & + \\ & + & \end{array}$

G
C
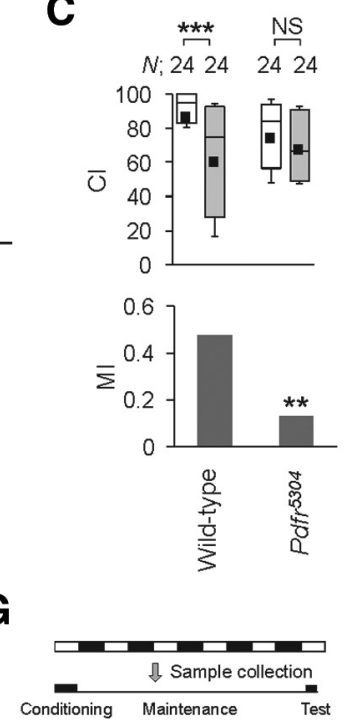

H
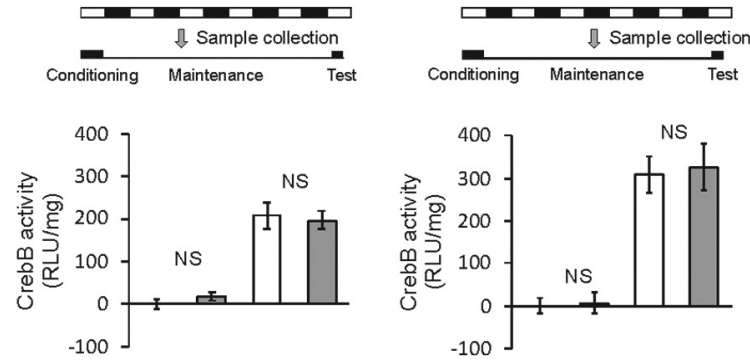

UAS-FLP

CRE>mCherry:: STOP $>$ IUC

$R 41 C 10(\alpha / \beta)$

$R 41 C 10(\alpha / \beta)$

Figure 6. Light-dependent transcriptional activity of CrebB in MB $\alpha / \beta$ neurons. $A$, Using UAS-FLP/hs-CrebB-B; CRE $>m C$ herry::STOP $>$ luc/R19B03 and UAS-FLPI+;CRE $>m C h e r r y:: S T O P>$ lucl $R 19 B 03$ flies, we examined whether the induction of the CrebB repressor CrebB-B inhibits CrebB activity. $N=9-11$ for each bar. In heat-shock treatment, males ( $3-4 \mathrm{~d}$ old) were kept at $32^{\circ} \mathrm{C}$ for $3 \mathrm{~d}$. Luc activity was measured immediately after the heat-shock treatment. Error bars indicate SEM. Nonparametric ANOVA (Kruskal-Wallis test) followed by post hoc analysis using the Steel-Dwass test was performed for multiple pairwise comparisons. Bars with the same letter indicate values that are not significantly different ( $p>0.05)$. B, The $($ rebB activity in MB $\alpha / \beta$ or $\gamma$ neurons was measured in LD or DD using MB $\alpha / \beta$-GAL4 (R41C10) or MB $\gamma$-GAL4 (R55D03). Samples were prepared between ZT 0 and ZT $2 . N=8-13$ in each bar. Error bars indicate SEM. One-way ANOVA followed by post hoc analysis using Scheffe's test was used [one-way ANOVA, $F_{(4,50)}=31.697, p<0.0001 ;$ Scheffe's multiple comparisons: (1) vs (2), $p<0.0001 ;(1)$ vs (3), $p=0.0169 ;(1)$ vs (4), $p<0.0001$; (1) vs (5), $p<0.0001$; (2) vs (3), $p<0.0001$; (2) vs (4), $p=0.9427 ;(2)$ vs (5), $p=0.5709 ;$ (3) vs (4), $p=0.0039 ;(3)$ vs (5), $p=0.0075 ;(4)$ vs (5), $p=0.9820$. Bars with the same letter indicate values that are not significantly different $(p>0.05)$. C, Memory on Day 5 in wild-type and $P d f r^{5304}$ flies. The Mann-Whitney $U$ test was used for comparisons of $C l$. The permutation test with 10,000 random permutations was used for comparisons of MI. For MI, asterisks indicate a comparison between wild-type and $P d f f^{5340}$. ${ }^{* *} p<0.01$, ${ }^{* * *} p<0.001$. NS, not significant; $N$, sample size in each box. D, UAS-FLP/R61G12-LexA; CRE $>m$ Cherry::STOP >luc LexAop-TrpA1/R41C10 and UAS-FLP/R61G12-LexA; CRE $>m$ Cherry::STOP $>$ Iuc /R41C10 flies were used. N = 5-6 for each bar. For activation of Pdf neurons by TrpA1, males ( $3-5 \mathrm{~d}$ old $)$ were kept at $32^{\circ} \mathrm{C}$ for $2 \mathrm{~d}$ in DD. Error bars indicate SEM. Student's $t$ test was used. ${ }^{* *} p<0.01$. $\boldsymbol{E}, \boldsymbol{F}$, The conditioning-dependent CrebB activity in $\mathrm{MB} \alpha / \beta$ or $\gamma$ neurons was measured immediately after $7 \mathrm{~h}$ conditioning $(\boldsymbol{E})$ and on Day $1(\mathrm{ZT} 0-2)$ after $7 \mathrm{~h}$ conditioning $(\boldsymbol{F})$. Student's $t$ test was used. ${ }^{*} p<0.05,{ }^{* *} p<0.01$. $N=$ 6-9 in each bar. $\mathbf{G}, \boldsymbol{H}$, The conditioning-dependent CrebB activity in MB $\alpha / \beta$ was measured at ZT $0-2(\boldsymbol{G})$ and ZT $16-18(\boldsymbol{H})$ on Day 2 after 7 h conditioning. Student's $t$ test was used. $N=9-10$ in each bar. $\boldsymbol{E}-\boldsymbol{H}$, White bars indicate naïve males, and gray bars indicate conditioned males.

arousal, geotactic behavior, rival-induced prolonged mating, and sex pheromone biosynthesis (Renn et al., 1999; Helfrich-Förster et al., 2000; Mertens et al., 2005; Kim et al., 2013; Krupp et al., 2013). Here, we found that Drosophila has a light-dependent memory maintenance system regulated by Pdf signaling. Pdf expression was essential for LTM maintenance in LD (Fig. 3). LTM was impaired when flies were kept in DD for $2 \mathrm{~d}$ after $7 \mathrm{~h}$ conditioning (Fig. 1), and the activation of Pdf neurons was sufficient to restore the LTM in DD (Fig. 2). Moreover, the electrical silencing of Pdf neurons impaired LTM in LD (Fig. 2). Considering that light activates Pdf neurons (Sheeba et al., 2008; Fogle et al., 2011; Ni et al., 2017), it is most likely that light-inducible Pdf released from Pdf neurons regulates LTM maintenance. We further confirmed that Pdfr expression is necessary for the light-driven transcription through CrebB, which is essential for LTM maintenance (Figs. 5, 6). Together, our study shows that this light-dependent transcription sys- tem in $\mathrm{MB} \alpha / \beta$ neurons via the Pdf signaling pathway regulates LTM maintenance in Drosophila.

Because Pdfr activation increases cAMP levels (Hyun et al., 2005; Mertens et al., 2005), Pdfr activation likely increases intracellular CREB activity. Previous studies using Pdfr-GAL4 lines or an anti-Pdfr antibody did not indicate Pdfr expression in MBs (Mertens et al., 2005; Im and Taghert, 2010). However, a recent study using RNA sequencing revealed that $P d f r$ is expressed in MBs (Crocker et al., 2016). Thus, it is possible that activated Pdfr in $\mathrm{MB} \alpha / \beta$ neurons directly enhances CREB activity in the same MB neurons (Fig. 7). Alternatively, Pdfr in non-MB neurons (e.g., dopaminergic neurons projecting to the MBs) may indirectly modify CREB activity in $\mathrm{MB} \alpha / \beta$ neurons (Fig. 7).

Drosophila has three light-sensing organs: the compound eyes, ocelli, and Hofbauer-Buchner (H-B) eyelets. The compound eyes play key roles in light entrainment of the circadian clock (Helfrich-Förster et al., 2002), and l-LNvs receive visual informa- 


\section{Non-MB neurons}
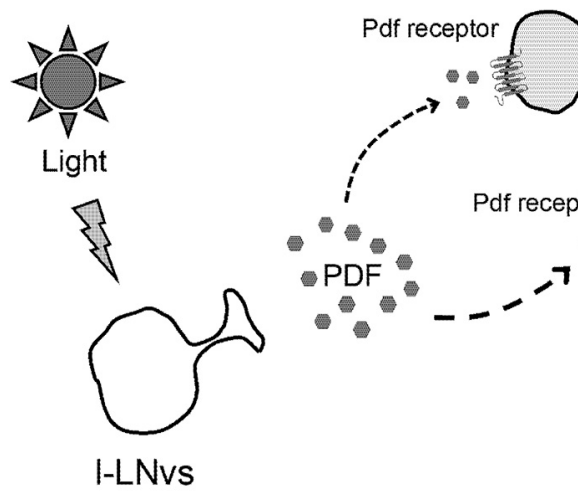

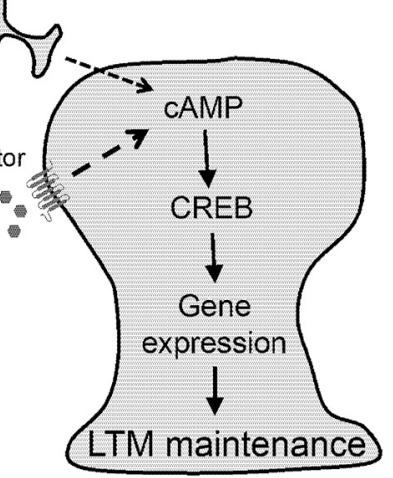

$\mathrm{MB} \alpha / \beta$ neurons

Figure 7. Possible model of light-dependent LTM maintenance. Light-dependent transcription of CREB via Pdf/Pdf receptor signaling is essential for LTM maintenance. Light-dependent Pdf release may induce activation of Pdf receptor leading to CAMP production in $\mathrm{MB} \alpha / \beta$ neurons. In addition, non-MB neurons with Pdfreceptor may also contribute to cAMP production in $\mathrm{MB} \alpha / \beta$ neurons.

tion via the compound eyes (Muraro and Ceriani, 2015). The $\mathrm{H}-\mathrm{B}$ eyelets are also important for circadian photoreception, and axons of $\mathrm{H}-\mathrm{B}$ eyelet photoreceptors project to the circadian pacemaker neurons including l-LNvs (Li et al., 2018). In addition, light directly activates Pdf neurons through the brain photoreceptors Rhodopsin 7 and Cryptochrome (Sheeba et al., 2008; Fogle et al., 2011; Ni et al., 2017). Although light input pathways associated with light-dependent LTM maintenance remain unclear, the photoactivation of light-sensing organs and/or Pdf neurons will trigger lightdependent LTM maintenance in Drosophila.

The targeted expression of a CrebB repressor in $\mathrm{MB} \alpha / \beta$ neurons during $7 \mathrm{~h}$ conditioning impaired LTM, as was observed in that in MB $\gamma$ neurons (Fig. 5), indicating that CrebB-dependent transcription in both $\mathrm{MB} \alpha / \beta$ and $\gamma$ neurons is necessary for memory consolidation. In addition, the targeted expression of a CrebB repressor in $\mathrm{MB} \alpha / \beta$ neurons during the memory maintenance phase also impaired LTM (Fig. 5), whereas, that in MB $\gamma$ neurons did not (Fig. 5). It has been reported that MB $\gamma$ neurons are necessary for $1 \mathrm{~d}$ memory of courtship conditioning (Krüttner et al., 2015). Thus, consolidated courtship memory may last in $\mathrm{MB} \gamma$ neurons only for $1-2 \mathrm{~d}$ at most. In contrast to the MB $\gamma$ neurons, the light-dependent activation of CrebB transcription was evident in $\mathrm{MB} \alpha / \beta$ neurons, indicating that $5 \mathrm{~d}$ memory is maintained in $\mathrm{MB} \alpha / \beta$ neurons through the $\mathrm{Pdf} / \mathrm{Pdfr} / \mathrm{CrebB}$ pathway in LD. Thus, long-lasting LTM ( $>1 \mathrm{~d}$ memory) seems to be established and stored in $\mathrm{MB} \alpha / \beta$ neurons.

When flies were kept in DD for $2 \mathrm{~d}$, LTM was impaired (Fig. 1) and the CrebB activity in $\mathrm{MB} \alpha / \beta$ neurons was severely attenuated (Fig. 6). However, DD for only $1 \mathrm{~d}$ was not sufficient to impair LTM (Fig. 1) but it reduced CrebB activity by 50\% (Fig. 6). Thus, when the CrebB activity in $\mathrm{MB} \alpha / \beta$ neurons is severely attenuated, LTM maintenance may break down. Pdf/Pdfr signaling should play a role in the regulation of the CrebB activity in MB $\alpha / \beta$ neurons because a $P d f r$-null mutation severely attenuated the CrebB activity (Fig. 6). However, it remains possible that signaling pathways other than the Pdf/Pdfr signaling pathway also contribute to the CrebB activity in $\mathrm{MB} \alpha / \beta$ neurons.

In contrast to LTM maintenance, LTM formation does not require light (Fig. 1). This finding is consistent with a previous report that STM after $1 \mathrm{~h}$ conditioning under a dim red light, which blocks visual input, remains intact (Joiner and Griffith,

1997). In this study, we found that CrebB activity during conditioning was necessary for memory consolidation (Fig. 5). Because flies were able to establish LTM when they were conditioned in darkness (Fig. 1), light-independent CrebB transcription in MBs plays an important role in memory consolidation. Unlike memory consolidation, LTM maintenance requires a light-dependent transcription system in $\mathrm{MB} \alpha / \beta$ neurons via the Pdf signaling pathway. In naive males, light can also increase the CrebB transcription activity in $\mathrm{MB} \alpha / \beta$ neurons (Fig. 6). This light-driven transcription system may play a role in innate brain functions other than LTM maintenance but does not provide proteins required for LTM maintenance. If this is the case, how are proteins required for LTM maintenance synthesized only in conditioned males? Although it remains unclear, we hypothesize that repetitive exposure to stressors during courtship conditioning may trigger the change in the target genes of CrebB in such a way that $\mathrm{MB} \alpha / \beta$ neurons produce gene products that are required for maintaining consolidated LTM in a courtship conditioning-dependent manner.

This study provided novel implications on how LTM consolidation and maintenance molecularly overlap or are distinct. We demonstrated that $1 \mathrm{~d}$ memory in $P d f^{01}$ mutant flies is intact but $2 \mathrm{~d}$ memory is impaired (Fig. $3 E$ ). We also showed that Pdf signaling enhances CREB activity and is required for LTM only after LTM is formed and stabilized. Together, our results indicate that CREB is necessary for both consolidation and maintenance phases, but how CREB is activated is different between these two memory phases. In the consolidation phase, multiple sensory inputs during conditioning trigger CREB activation. In contrast, in the maintenance phase, initial sensory signals disappear and can no longer activate CREB, but environmental light to which flies are regularly exposed to under normal conditions activates CREB through Pdf signaling. Thus, we may be able to molecularly distinguish maintenance from consolidation on the basis of whether light and Pdf are required for CREB activation. It is important to determine whether this finding can be applied to other memory paradigms in Drosophila such as olfactory LTM.

In nature, animals learn much from their experience throughout the day. Through their experience, LTM is formed and maintained for a long period. If environmental light, which is available daily to all animals in nature, can be used for transcriptional activation in the brain, such a light-driven transcription system is considered reasonable and effective for continually providing de novo protein synthesis required for LTM maintenance. When diurnal Nile grass rats were housed under dim LD cycles, $24 \mathrm{~h}$ spatial memory was impaired, and this lighting condition inhibited the expression of brain-derived neurotrophic factors and the dendritic spine density in the hippocampus (Soler et al., 2018). Although it is not clarified whether the rapid forgetting in the Nile grass rats under dim LD cycles results from the inhibition of de novo protein synthesis required for LTM maintenance, lightdependent de novo protein synthesis in the memory center may be conserved in many animal species. As is observed in Drosophila, in mammals, repeated exposure to stressors also induces a long-lasting reduction of male sexual motivation (Hawley et al., 
2011; Hawley et al., 2013). It is thus interesting to examine whether environmental light and the evolutionarily conserved memory modulator CREB also play critical roles in the maintenance of such depressed sexual motivation in mammals including humans.

\section{References}

Alberini CM (2009) Transcription factors in long-term memory and synaptic plasticity. Physiol Rev 89:121-145.

Altimus CM, Güler AD, Villa KL, McNeill DS, Legates TA, Hattar S (2008) Rods-cones and melanopsin detect light and dark to modulate sleep independent of image formation. Proc Natl Acad Sci US A 105: 19998-20003.

Bekinschtein P, Cammarota M, Igaz LM, Bevilaqua LR, Izquierdo I, Medina JH (2007) Persistence of long-term memory storage requires a late protein synthesis- and BDNF-dependent phase in the hippocampus. Neuron 53:261-277.

Cajochen C, Frey S, Anders D, Späti J, Bues M, Pross A, Mager R, Wirz-Justice A, Stefani O (2011) Evening exposure to a light-emitting diodes (LED)backlit computer screen affects circadian physiology and cognitive performance. J Appl Physiol 110:1432-1438.

Chellappa SL, Ly JQ, Meyer C, Balteau E, Degueldre C, Luxen A, Phillips C, Cooper HM, Vandewalle G (2014) Photic memory for executive brain responses. Proc Natl Acad Sci U S A 111:6087-6091.

Crocker A, Sehgal A (2010) Genetic analysis of sleep. Genes Dev 24:12201235.

Crocker A, Guan XJ, Murphy CT, Murthy M (2016) Cell-type-specific transcriptome analysis in the Drosophila mushroom body reveals memoryrelated changes in gene expression. Cell Rep 15:1580-1596.

Davis RL (2011) Traces of Drosophila memory. Neuron 70:8-19.

Donlea JM, Thimgan MS, Suzuki Y, Gottschalk L, Shaw PJ (2011) Inducing sleep by remote control facilitates memory consolidation in Drosophila. Science 332:1571-1576.

Dubnau J, Chiang AS (2013) Systems memory consolidation in Drosophila. Curr Opin Neurobiol 23:84-91.

Fernandez DC, Fogerson PM, Lazzerini Ospri L, Thomsen MB, Layne RM, Severin D, Zhan J, Singer JH, Kirkwood A, Zhao H, Berson DM, Hattar S (2018) Light affects mood and learning through distinct retina-brain pathways. Cell 175:71-84.e18.

Fioriti L, Myers C, Huang YY, Li X, Stephan JS, Trifilieff P, Colnaghi L, Kosmidis S, Drisaldi B, Pavlopoulos E, Kandel ER (2015) The persistence of hippocampal-based memory requires protein synthesis mediated by the prion-like protein CPEB3. Neuron 86:1433-1448.

Fogle KJ, Parson KG, Dahm NA, Holmes TC (2011) CRYPTOCHROME is a blue-light sensor that regulates neuronal firing rate. Science 331:14091413.

Ganguly-Fitzgerald I, Donlea J, Shaw PJ (2006) Waking experience affects sleep need in Drosophila. Science 313:1775-1781.

Griffith LC, Ejima A (2009) Courtship learning in Drosophila melanogaster: diverse plasticity of a reproductive behavior. Learn Mem 16:743-750.

Hamada FN, Rosenzweig M, Kang K, Pulver SR, Ghezzi A, Jegla TJ, Garrity PA (2008) An internal thermal sensor controlling temperature preference in Drosophila. Nature 454:217-220.

Hawley W, Grissom E, Keskitalo L, Hastings T, Dohanich G (2011) Sexual motivation and anxiety-like behaviors of male rats after exposure to a trauma followed by situational reminders. Physiol Behav 102:181-187.

Hawley WR, Grissom EM, Belkin MN, James TF, Dohanich GP (2013) Decreased sexual motivation and heightened anxiety in male Long-Evans rats are correlated with the memory for a traumatic event. Arch Sex Behav 42:659-668.

Helfrich-Förster C, Täuber M, Park JH, Muhlig-Versen M, Schneuwly S, Hofbauer A (2000) Ectopic expression of the neuropeptide pigmentdispersing factor alters behavioral rhythms in Drosophila melanogaster. J Neurosci 20:3339-3353.

Helfrich-Förster C, Edwards T, Yasuyama K, Wisotzki B, Schneuwly S, Stanewsky R, Meinertzhagen IA, Hofbauer A (2002) The extraretinal eyelet of Drosophila: development, ultrastructure, and putative circadian function. J Neurosci 22:9255-9266.

Hirano Y, Ihara K, Masuda T, Yamamoto T, Iwata I, Takahashi A, Awata H, Nakamura N, Takakura M, Suzuki Y, Horiuchi J, Okuno H, Saitoe M (2016) Shifting transcriptional machinery is required for long-term memory maintenance and modification in Drosophila mushroom bodies. Nat Commun 7:13471.

Huber R, Hill SL, Holladay C, Biesiadecki M, Tononi G, Cirelli C (2004) Sleep homeostasis in Drosophila melanogaster. Sleep 27:628-639.

Hyun S, Lee Y, Hong ST, Bang S, Paik D, Kang J, Shin J, Lee J, Jeon K, Hwang S, Bae E, Kim J (2005) Drosophila GPCR han is a receptor for the circadian clock neuropeptide PDF. Neuron 48:267-278.

Im SH, Taghert PH (2010) PDF receptor expression reveals direct interactions between circadian oscillators in Drosophila. J Comp Neurol 518:1925-1945.

Ishimoto H, Sakai T, Kitamoto T (2009) Ecdysone signaling regulates the formation of long-term courtship memory in adult Drosophila melanogaster. Proc Natl Acad Sci U S A 106:6381-6386.

Joiner MA, Griffith LC (1997) CaM kinase II and visual input modulate memory formation in the neuronal circuit controlling courtship conditioning. J Neurosci 17:9384-9391.

Kandel ER (2012) The molecular biology of memory: cAMP, PKA, CRE, CREB-1, CREB-2, and CPEB. Mol Brain 5:14.

Keleman K, Vrontou E, Krüttner S, Yu JY, Kurtovic-Kozaric A, Dickson BJ (2012) Dopamine neurons modulate pheromone responses in Drosophila courtship learning. Nature 489:145-149.

Kim WJ, Jan LY, Jan YN (2013) A PDF/NPF neuropeptide signaling circuitry of male Drosophila melanogaster controls rival-induced prolonged mating. Neuron 80:1190-1205.

Koemans TS, Oppitz C, Donders RAT, van Bokhoven H, Schenck A, Keleman K, Kramer JM (2017) Drosophila courtship conditioning as a measure of learning and memory. J Vis Exp 124:e55808.

Krupp JJ, Billeter JC, Wong A, Choi C, Nitabach MN, Levine JD (2013) Pigment-dispersing factor modulates pheromone production in clock cells that influence mating in Drosophila. Neuron 79:54-68.

Krüttner S, Traunmüller L, Dag U, Jandrasits K, Stepien B, Iyer N, Fradkin LG, Noordermeer JN, Mensh BD, Keleman K (2015) Synaptic Orb2A bridges memory acquisition and late memory consolidation in Drosophila. Cell Rep 11:1953-1965.

Kume K, Kume S, Park SK, Hirsh J, Jackson FR (2005) Dopamine is a regulator of arousal in the fruit fly. J Neurosci 25:7377-7384.

Lee SS, Ding Y, Karapetians N, Rivera-Perez C, Noriega FG, Adams ME (2017) Hormonal signaling cascade during an early-adult critical period required for courtship memory retention in Drosophila. Curr Biol 27: 2798-2809.e3.

Lee YS, Bailey CH, Kandel ER, Kaang BK (2008) Transcriptional regulation of long-term memory in the marine snail Aplysia. Mol Brain 1:3.

LeGates TA, Altimus CM, Wang H, Lee HK, Yang S, Zhao H, Kirkwood A, Weber ET, Hattar S (2012) Aberrant light directly impairs mood and learning through melanopsin-expressing neurons. Nature 491:594-598.

Li MT, Cao LH, Xiao N, Tang M, Deng B, Yang T, Yoshii T, Luo DG (2018) Hub-organized parallel circuits of central circadian pacemaker neurons for visual photoentrainment in Drosophila. Nat Commun 9:4247.

Majumdar A, Cesario WC, White-Grindley E, Jiang H, Ren F, Khan MR, Li L, Choi EM, Kannan K, Guo F, Unruh J, Slaughter B, Si K (2012) Critical role of amyloid-like oligomers of Drosophila Orb2 in the persistence of memory. Cell 148:515-529.

Margulies C, Tully T, Dubnau J (2005) Deconstructing memory in Drosophila. Curr Biol 15:R700-R713.

McGuire SE, Le PT, Osborn AJ, Matsumoto K, Davis RL (2003) Spatiotemporal rescue of memory dysfunction in Drosophila. Science 302:17651768.

Mertens I, Vandingenen A, Johnson EC, Shafer OT, Li W, Trigg JS, De Loof A, Schoofs L, Taghert PH (2005) PDF receptor signaling in Drosophila contributes to both circadian and geotactic behaviors. Neuron 48:213-219.

Muraro NI, Ceriani MF (2015) Acetylcholine from visual circuits modulates the activity of arousal neurons in Drosophila. J Neurosci 35:1631516327.

Ni JD, Baik LS, Holmes TC, Montell C (2017) A rhodopsin in the brain functions in circadian photoentrainment in Drosophila. Nature 545:340 344.

Pan Y, Baker BS (2014) Genetic identification and separation of innate and experience-dependent courtship behaviors in Drosophila. Cell 156: $236-248$

Qiu J, Hardin PE (1996) per mRNA cycling is locked to lights-off under photoperiodic conditions that support circadian feedback loop function. Mol Cell Biol 16:4182-4188. 
Renn SC, Park JH, Rosbash M, Hall JC, Taghert PH (1999) A pdf neuropeptide gene mutation and ablation of PDF neurons each cause severe abnormalities of behavioral circadian rhythms in Drosophila. Cell 99:791-802.

Sakai T, Tamura T, Kitamoto T, Kidokoro Y (2004) A clock gene, period, plays a key role in long-term memory formation in Drosophila. Proc Natl Acad Sci U S A 101:16058-16063.

Sakai T, Sato S, Ishimoto H, Kitamoto T (2012) Significance of the centrally expressed TRP channel painless in Drosophila courtship memory. Learn Mem 20:34-40.

Shan LL, Guo H, Song NN, Jia ZP, Hu XT, Huang JF, Ding YQ, RichterLevine G, Zhou QX, Xu L (2015) Light exposure before learning improves memory consolidation at night. Sci Rep 5:15578.

Sheeba V, Gu H, Sharma VK, O’Dowd DK, Holmes TC (2008) Circadianand light-dependent regulation of resting membrane potential and spontaneous action potential firing of Drosophila circadian pacemaker neurons. J Neurophysiol 99:976-988.

Shimada N, Inami S, Sato S, Kitamoto T, Sakai T (2016) Modulation of light-driven arousal by LIM-homeodomain transcription factor apterous in large PDF-positive lateral neurons of the Drosophila brain. Sci Rep 6:37255.
Siegel RW, Hall JC (1979) Conditioned responses in courtship behavior of normal and mutant Drosophila. Proc Natl Acad Sci U S A 76:3430-3434.

Soler JE, Robison AJ, Núñez AA, Yan L (2018) Light modulates hippocampal function and spatial learning in a diurnal rodent species: a study using male nile grass rat (Arvicanthis niloticus). Hippocampus 28:189-200.

Taghert PH, Hewes RS, Park JH, O’Brien MA, Han M, Peck ME (2001) Multiple amidated neuropeptides are required for normal circadian locomotor rhythms in Drosophila. J Neurosci 21:6673-6686.

Tanenhaus AK, Zhang J, Yin JC (2012) In vivo circadian oscillation of dCREB 2 and NF- $\kappa$ B activity in the Drosophila nervous system. PloS One 7:e45130.

Tully T, Preat T, Boynton SC, Del Vecchio M (1994) Genetic dissection of consolidated memory in Drosophila. Cell 79:35-47.

Vandewalle G, Maquet P, Dijk DJ (2009) Light as a modulator of cognitive brain function. Trends Cogn Sci 13:429-438.

Yin JC, Tully T (1996) CREB and the formation of long-term memory. Curr Opin Neurobiol 6:264-268.

Yin JC, Wallach JS, Del Vecchio M, Wilder EL, Zhou H, Quinn WG, Tully T (1994) Induction of a dominant negative CREB transgene specifically blocks long-term memory in Drosophila. Cell 79:49-58. 